\title{
A Variational Formulation for Dirac Operators in Bounded Domains: Applications to Spectral Geometric Inequalities
}

\section{Pedro R. S.Antunes ${ }^{1,2}$, RafaelD. Benguria ${ }^{3}$, VladimirLotoreichik ${ }^{4}$, ThomasOurmières-Bonafos ${ }^{5}$}

1 Secção de Matemática, Departamento de Ciências e Tecnologia, Universidade Aberta, Palácio Ceia, 1269001 Lisbon, Portugal. E-mail: prantunes@fc.ul.pt

2 Grupo de Física Matemática, Faculdade de Ciências, Universidade de Lisboa, Campo Grande, Edifício C6, 1749-016 Lisboa, Portugal.

3 Instituto de Física, Pontificia Universidad Católica de Chile, Avda. Vicuña Mackenna, 4860 Santiago, Chile. E-mail: rbenguri@fis.puc.cl

4 Department of Theoretical Physics, Nuclear Physics Institute, Czech Academy of Sciences, 25068 Řež, Czech Republic. E-mail: lotoreichik@ujf.cas.cz

5 CNRS, Centrale Marseille, I2M, Aix Marseille Univ, Marseille, France.

E-mail: thomas.ourmieres-bonafos@univ-amu.fr

Received: 23 March 2020 / Accepted: 13 January 2021

(C) The Author(s), under exclusive licence to Springer-Verlag GmbH, DE part of Springer Nature 2021

\begin{abstract}
We investigate spectral features of the Dirac operator with infinite mass boundary conditions in a smooth bounded domain of $\mathbb{R}^{2}$. Motivated by spectral geometric inequalities, we prove a non-linear variational formulation to characterize its principal eigenvalue. This characterization turns out to be very robust and allows for a simple proof of a Szegó type inequality as well as a new reformulation of a Faber-Krahn type inequality for this operator. The paper is complemented with strong numerical evidences supporting the existence of a Faber-Krahn type inequality.
\end{abstract}

\section{Contents}

1. Introduction

2. Preliminaries

3. Maximal Wirtinger Operators

4. Bergman and Hardy Spaces on $\Omega$

5. Variational Characterization of the Principal Eigenvalue

6. Geometric Upper Bounds on the Spectral Gap

7. About the Faber-Krahn Conjecture

8. Numerics

\section{Introduction}

1.1. Motivations and state of the art. In the past few years there has been a growing interest in the study of Dirac operators among the mathematical physics community; the main reason being that low-energy electrons in a single-layered sheet of graphene are driven by an effective Hamiltonian being a two-dimensional massless Dirac operator.

\begin{tabular}{|l|l|l|l|l|l|}
\hline $\mathbf{2} 2 \mathbf{2 0}$ & $\mathbf{3 9 5 9}$ & $\mathbf{B}$ & $\begin{array}{l}\text { Dispatch: 30/1/2021 } \\
\text { Total pages: 38 } \\
\text { Disk Received } \\
\text { Disk Used } \square\end{array}$ & $\begin{array}{l}\text { Journal: Commun. Math. Phys. } \\
\text { Not Used } \square \\
\text { Corrupted } \square \\
\text { Mismatch } \square\end{array}$ \\
\hline Jour. No & Ms. No.
\end{tabular}


Various mathematical studies have been undertaken, starting with a rigorous mathematical derivation of such Hamiltonians, see e.g. [19] for the effective Hamiltonian derivation or $[3,8,30,38]$ for the justification of the so-called infinite mass boundary conditions. Many properties of such operators have been investigated as their self-adjointness in bounded domains with specified boundary conditions or coupled with the so-called $\delta$-interactions, see $[9,11]$. Let us also mention recent works on spectral properties and asymptotics of Dirac-type operators in specific asymptotic regimes (see [4,23]).

In this work, we are interested in finding geometrical bounds on the eigenvalues of one of the simplest Dirac operator relevant in physics: the two-dimensional massless Dirac operator with infinite mass boundary conditions.

To set the stage, let $\Omega \subset \mathbb{R}^{2}$ be a $C^{\infty}$ simply connected and bounded domain and let $n=\left(n_{1}, n_{2}\right)^{\top}$ be the outward pointing normal field on $\partial \Omega$. The Dirac operator with infinite mass boundary conditions in $L^{2}\left(\Omega, \mathbb{C}^{2}\right)$ is defined as

$$
\begin{aligned}
D^{\Omega} & :=\left(\begin{array}{cc}
0 & -2 \mathrm{i} \partial_{z} \\
-2 \mathrm{i} \partial_{\bar{z}} & 0
\end{array}\right), \\
\operatorname{dom}\left(D^{\Omega}\right) & :=\left\{u=\left(u_{1}, u_{2}\right)^{\top} \in H^{1}\left(\Omega, \mathbb{C}^{2}\right): u_{2}=\operatorname{in} u_{1} \text { on } \partial \Omega\right\},
\end{aligned}
$$

where we have set $\mathbf{n}:=n_{1}+\mathrm{i} n_{2}$ and with the Wirtinger operators defined as usual by

$$
\partial_{z}=\frac{1}{2}\left(\partial_{1}-\mathrm{i} \partial_{2}\right), \quad \partial_{\bar{z}}=\frac{1}{2}\left(\partial_{1}+\mathrm{i} \partial_{2}\right) \text {. }
$$

The Dirac operator with infinite mass boundary conditions $D^{\Omega}$ is known to be selfadjoint (see [11, Thm. 1.1.]), moreover its spectrum is symmetric with respect to the origin and constituted of eigenvalues of finite multiplicity satisfying

$$
\cdots \leq-E_{k}(\Omega) \leq \cdots \leq-E_{1}(\Omega)<0<E_{1}(\Omega) \leq \cdots \leq E_{k}(\Omega) \leq \cdots .
$$

In the recent paper [12], the following geometrical lower bound is obtained

$$
E_{1}(\Omega) \geq \sqrt{\frac{2 \pi}{|\Omega|}},
$$

where $|\Omega|$ denotes the area of the domain $\Omega$. However, this lower bound is never attained among Euclidean domains and by analogy with the famous Faber-Krahn inequality [18, 26], a natural conjecture for the optimal lower-bound is the following.

\section{Conjecture 1. There holds}

$$
E_{1}(\Omega) \geq \sqrt{\frac{\pi}{|\Omega|}} E_{1}(\mathbb{D}),
$$

where $\mathbb{D}$ is the unit disk. There is equality in the above inequality if and only if $\Omega$ is a disk.

Remark 2. As explained in [12, Remark 2] (see also [28, Appendix]), the eigenstructure of the unit disk is explicit. Indeed, $E_{1}(\mathbb{D}) \simeq 1.435 \ldots$ is the first non-negative root of the equation $J_{0}(E)=J_{1}(E)$ where $J_{0}$ and $J_{1}$ are the Bessel functions of the first kind of order 0 and of order 1 , respectively. Moreover, an associated eigenfunction is given for $\left(x_{1}, x_{2}\right) \in \mathbb{D}$ by

$$
\left(\begin{array}{c}
J_{0}\left(E_{1}(\mathbb{D})|x|\right) \\
\mathrm{i} \frac{x_{1}+\mathrm{i} x_{2}}{|x|} J_{1}\left(E_{1}(\mathbb{D})|x|\right)
\end{array}\right) .
$$

\begin{tabular}{|l|l|l|l|l|l|}
\hline $\mathbf{2} 20$ & $\mathbf{3 9 5 9}$ & $\mathbf{B}$ & $\begin{array}{l}\text { Dispatch: 30/1/2021 } \\
\text { Total pages: 38 } \\
\text { Disk Received } \\
\text { Disk Used } \square\end{array}$ & $\begin{array}{l}\text { Journal: Commun. Math. Phys. } \\
\text { Not Used } \square \\
\text { Corrupted } \square \\
\text { Mismatch } \square\end{array}$ \\
\hline Jour. No & Ms. No.
\end{tabular}


Conjecture 1 motivated part of this paper and is still an open question. However, in Sect. 8 we provide strong numerical evidences supporting it and in Sect. 7 we show how Conjecture 1 is intimately connected to the famous Bossel-Daners inequality for the Robin Laplacian (see $[13,15])$.

The quest for a geometrical upper-bound has also attracted attention recently as for instance in [28]. In this work, the given geometrical upper-bound is sharp in the sense that it is an equality if and only if the considered domain is a disk. Nevertheless, this upper-bound depends in a complicated fashion on different geometrical parameters and may be hard to compute in practice.

Let us also mention that similar questions are dealt with within the differential geometry literature for lower bounds and upper bounds for Dirac operators on spin-manifolds (see for instance $[1,6,7,33]$ ).

One of the main results of this paper is the following theorem which gives a geometrical upper-bound in terms of simple geometric quantities: $|\Omega|$ - the area of $\Omega,|\partial \Omega|$ - the perimeter of $\Omega$ as well as $r_{i}$-the inradius of $\Omega$.

Theorem 3. Let $\Omega \subset \mathbb{R}^{2}$ be a $C^{\infty}$ simply connected and bounded domain. There holds

$$
E_{1}(\Omega) \leq \frac{|\partial \Omega|}{\left(\pi r_{i}^{2}+|\Omega|\right)} E_{1}(\mathbb{D}),
$$

with equality if and only if $\Omega$ is a disk.

The proof is by combining a new variational characterization of $E_{1}(\Omega)$, inspired by min-max techniques for operators with gaps introduced in [16,22] and the classical proof of Szegó about the eigenvalues of membranes of fixed area [39] (see also the recent work [36] on operators with gaps).

It turns out that this new variational characterization is of interest by itself because it also allows for numerical simulations and we believe that it could be an adequate starting point to prove Conjecture 1 as discussed further on in Sect. 7. To introduce it, consider the quadratic form

$$
\begin{aligned}
q_{E, 0}^{\Omega}(u) & :=4 \int_{\Omega}\left|\partial_{\bar{z}} u\right|^{2} d x-E^{2} \int_{\Omega}|u|^{2} d x+E \int_{\partial \Omega}|u|^{2} d s, \\
\operatorname{dom}\left(q_{E, 0}^{\Omega}\right) & :=C^{\infty}(\bar{\Omega}, \mathbb{C}) .
\end{aligned}
$$

For $E>0, q_{E, 0}^{\Omega}$ is bounded below with dense domain and we consider $q_{E}^{\Omega}$ the closure in $L^{2}(\Omega)$ of $q_{E, 0}^{\Omega}$. Then, we define the first min-max level

$$
\mu^{\Omega}(E):=\inf _{u \in \operatorname{dom}\left(q_{E}^{\Omega}\right) \backslash\{0\}} \frac{4 \int_{\Omega}\left|\partial_{\bar{z}} u\right|^{2} d x-E^{2} \int_{\Omega}|u|^{2} d x+E \int_{\partial \Omega}|u|^{2} d s}{\int_{\Omega}|u|^{2} d x} .
$$

The second main result of this paper is the following non-linear variational characterization of $E_{1}(\Omega)$.

Theorem 4. $E>0$ is the first non-negative eigenvalue of $D^{\Omega}$ if and only if $\mu^{\Omega}(E)=0$. The advantage of the quadratic form $q_{E}^{\Omega}$ is two-fold. First, functions in the considered variational space are now scalar valued and, second, the infinite mass boundary conditions does not appear in the variational formulation. However, the first drawback is that dom $\left(q_{E}^{\Omega}\right)$ contains the Hardy space $\mathcal{H}_{\mathrm{h}}^{2}(\Omega)$, constituted of holomorphic functions with

ニ


traces in $L^{2}(\partial \Omega)$. In particular, $\operatorname{dom}\left(q_{E}^{\Omega}\right)$ is not a usual Sobolev space and a special care is needed in order to prove Theorem 4 . In particular, it requires a precise description of the domain dom $\left(q_{E}^{\Omega}\right)$ as well as the domain of the associated self-adjoint operator via Kato's first representation theorem (see [25, Chap. VI, Thm. 2.1]). It is done using convolution operators reminiscent of what is done in [5,31], elliptic regularity properties of the maximal Wirtinger operators as well as using Cauchy singular integral operators on $\partial \Omega$, seen as periodic pseudo-differential operators.

Theorem 4 is reminiscent of $[16,17]$, where a similar strategy is used to deal with the Dirac-Coulomb operator. To our knowledge, this is the first time this idea is extended to boundary value problems and now, we describe its heuristic.

Let $(u, v)^{\top} \in \operatorname{dom}\left(D^{\Omega}\right)$ be an eigenfunction associated with the eigenvalue $E>0$. In $\Omega$, the eigenvalue equation reads

$$
-2 \mathrm{i} \partial_{z} v=E u, \quad-2 \mathrm{i} \partial_{\bar{z}} u=E v .
$$

If we assume that this identity is true up to the boundary $\partial \Omega$, we obtain the following boundary condition for $u$ :

$$
\overline{\mathbf{n}} \partial_{\bar{z}} u+\frac{E}{2} u=0 \text { on } \partial \Omega
$$

Now, Eq. (4) gives

$$
-4 \partial_{z} \partial_{\bar{z}} u=E^{2} u \text { in } \Omega \text {. }
$$

Hence, a weak formulation is obtained taking the scalar product by $u$, integrating by parts and taking into account the boundary condition (5). This formally gives $q_{E}^{\Omega}(u)=0$ and this is the reason for introducing the quadratic form $q_{E}^{\Omega}$ in (2).

Let us add two remarks. The first one explains that (5)-(6) can be recast into a nonlinear eigenvalue problem for a Laplace operator with oblique boundary conditions. The second remark, explains how Theorem 4 could be extended to handle the next eigenvalues.

Remark 5. Note that (6) is an eigenvalue equation for the Laplace operator and reads $-\Delta u=E^{2} u$. The boundary condition (5) is a relation between the normal derivative, the tangential derivative and the value of the function on $\partial \Omega$. If we let $\mathbf{t}$ be the tangent field on $\partial \Omega$ such that $(\mathbf{n}, \mathbf{t})$ is a direct frame, the problem can be re-interpreted as an oblique problem

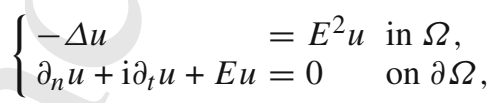

where $\partial_{n}$ and $\partial_{t}$ are the normal and tangential derivatives, respectively.

Note that Problem (7) is non-linear because the parameter $E>0$ appears both in the eigenvalue equation and in the boundary condition.

Remark 6. For $j \geq 1$, one can consider the $j$ th min-max level of $q_{E}^{\Omega}$ defined as

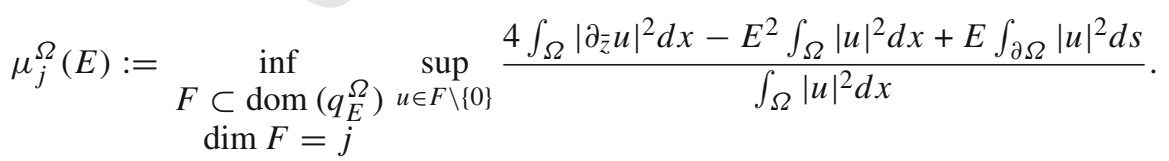

As in [16], Theorem 4 could be extended as follows: $E>0$ is the $j$ th non-negative eigenvalue of $D^{\Omega}$ if and only if $\mu_{j}^{\Omega}(E)=0$. We do not discuss it here because we are concerned only with the principal eigenvalue $E_{1}(\Omega)$.

\begin{tabular}{|l|l|l|l|l|l|}
\hline $\mathbf{2} 2 \mathbf{2 0}$ & $\mathbf{3 9 5 9}$ & $\mathbf{B}$ & $\begin{array}{l}\text { Dispatch: 30/1/2021 } \\
\text { Total pages: 38 } \\
\text { Disk Received } \\
\text { Disk Used } \square\end{array}$ & $\begin{array}{l}\text { Journal: Commun. Math. Phys. } \\
\text { Not Used } \square \\
\text { Corrupted } \\
\text { Mismatch } \square\end{array}$ \\
\hline Jour. No & Ms. No.
\end{tabular}


Finally, let us comment the hypothesis on $\Omega$. First, one would like to lower the smoothness hypothesis to be able to handle, for instance, Lipschitz domains. This is a natural question but there is no reason for the Dirac operator with infinite mass boundary to be self-adjoint on such a domain dom $\left(D^{\Omega}\right)$ (see the case of polygonal domains in [27]). Moreover, as part of the proof relies on pseudo-differential techniques, we prefer to keep the $C^{\infty}$ smoothness assumption on $\partial \Omega$ because it allows for a more efficient treatment of singular integral operators on the boundary. Second, the simply connectedness assumption may be an unnecessary hypothesis for Theorem 4 to hold. Nevertheless, we are not able to drop it in Theorem 3 because the proof relies on the Riemann mapping theorem to build an admissible test function for $q_{E}^{\Omega}$.

\subsection{Structure of the paper. In Sect. 2, we gather several results on Sobolev spaces on} $\partial \Omega$, periodic pseudo-differential operators on $\partial \Omega$ and deduce various mapping properties of the Cauchy singular integral operators.

Section 3 contains a description of the domain of the maximal Wirtinger operators. In particular, we discuss the existence of a trace operator for functions belonging to these domains and state a fundamental elliptic regularity result.

Section 4 deals with the description of the Bergman and Hardy spaces on $\Omega$ thanks to integral operators. This is done by introducing the Szegó projectors on the Sobolev spaces on the boundary $H^{s}(\partial \Omega)\left(s \in\left\{-\frac{1}{2}, 0, \frac{1}{2}\right\}\right)$. As a byproduct of this analysis we are able to describe explicitly the domains of the maximal Wirtinger operators.

Theorem 4 is proved in Sect. 5. We start by describing the domain of the quadratic form $q_{E}^{\Omega}$ in terms of the first-order Sobolev space $H^{1}(\Omega)$ and the Hardy space on $\Omega$. Then, the analysis is pushed forward to study the domain of the self-adjoint operator associated with $q_{E}^{\Omega}$ via Kato's first representation theorem (see [25, Chap. VI, Thm. 2.1]). Combining these tools, we prove Theorem 4.

Then, we apply Theorem 4 in Sect. 6 to prove Theorem 3. The proof is by adapting the well-known proof of Szegó [39] to our setting, constructing an adequate test function for the new variational formulation.

In Sect. 7, we show that Conjecture 1 can be reformulated and that it is related to the famous Bossel-Daners inequality.

We conclude in Sect. 8 illustrating by numerical experiments the validity of Conjecture 1 and several theoretical results discussed all along the paper.

\section{Preliminaries}

2.1. Sobolev spaces on $\partial \Omega$. In the following, $\mathbb{T}$ is the torus $\mathbb{T}:=\mathbb{R} / \mathbb{Z}, \mathcal{D}(\mathbb{T})=C^{\infty}(\mathbb{T})$ is the space periodic smooth functions on the torus $\mathbb{T}$ and $\mathcal{D}(\mathbb{T})^{\prime}$ the space of periodic distributions on the torus $\mathbb{T}$. Let $f \in \mathcal{D}(\mathbb{T})^{\prime}$ we define its Fourier coefficients using the duality pairing by

$$
\widehat{f}(n):=\left\langle f, e_{-n}\right\rangle_{\mathcal{D}(\mathbb{T})^{\prime}, \mathcal{D}(\mathbb{T})}, \quad e_{n}:=t \in \mathbb{T} \mapsto e^{2 \mathrm{i} \pi n t} .
$$

For $s \in \mathbb{R}$, the Sobolev space of order $s$ on $\mathbb{T}$ is defined as

$$
H^{s}(\mathbb{T}):=\left\{f \in \mathcal{D}(\mathbb{T})^{\prime}: \sum_{n=-\infty}^{+\infty}(1+|n|)^{2 s}|\widehat{f}(n)|^{2}<+\infty\right\} .
$$

\begin{tabular}{|l|l|l|l|l|l|}
\hline 220 & $\mathbf{3 9 5 9}$ & $\mathbf{B}$ & $\begin{array}{l}\text { Dispatch: 30/1/2021 } \\
\text { Total pages: 38 } \\
\text { Disk Received } \\
\text { Disk Used } \square\end{array}$ & $\begin{array}{l}\text { Journal: Commun. Math. Phys. } \\
\text { Not Used } \square \\
\text { Corrupted } \square \\
\text { Mismatch } \square\end{array}$ \\
\hline Jour. No
\end{tabular}


Set $\ell:=|\partial \Omega|$ and let $\gamma: \mathbb{R} /[0, \ell] \rightarrow \partial \Omega$ be a smooth arc-length parametrization of $\partial \Omega$. Consider the map

$$
U^{*}: \mathcal{D}(\mathbb{T}) \rightarrow \mathcal{D}(\partial \Omega), \quad\left(U^{*} g\right)(x):=\ell^{-1} g\left(\ell^{-1} \gamma^{-1}(x)\right), x \in \partial \Omega,
$$

where we have set $\mathcal{D}(\partial \Omega):=C^{\infty}(\partial \Omega)$. We define the map $U: \mathcal{D}(\partial \Omega)^{\prime} \rightarrow \mathcal{D}(\mathbb{T})^{\prime}$ as

$$
\langle U f, g\rangle_{\mathcal{D}(\mathbb{T})^{\prime}, \mathcal{D}(\mathbb{T})}:=\left\langle f, U^{*} g\right\rangle_{\mathcal{D}(\partial \Omega)^{\prime}, \mathcal{D}(\partial \Omega)} .
$$

The Sobolev space of order $s \in \mathbb{R}$ on $\partial \Omega$ is defined as

$$
H^{s}(\partial \Omega):=\left\{f \in \mathcal{D}(\partial \Omega)^{\prime}: U f \in H^{s}(\mathbb{T})\right\} .
$$

2.2. Periodic pseudo-differential operators. Let us start by defining periodic pseudodifferential operators on $\mathbb{T}$.

Definition 7. A linear operator $H$ on $C^{\infty}(\mathbb{T})$ is a periodic pseudo-differential operator on $\mathbb{T}$ if there exists $h: \mathbb{T} \times \mathbb{Z} \rightarrow \mathbb{C}$ such that:

1. for all $n \in \mathbb{Z}, h(\cdot, n) \in C^{\infty}(\mathbb{T})$,

2. $H$ acts as $H f=\sum_{n \in \mathbb{Z}} h(\cdot, n) \widehat{f}(n) e_{n}$,

3. there exists $\alpha \in \mathbb{R}$ such that for all $p, q \in \mathbb{N}_{0}$ there exists $c_{p, q}>0$ such that there holds

$$
\left|\left(\frac{d^{p}}{d t^{p}}\left(\omega^{q} h\right)\right)(t, n)\right| \leq c_{p, q}(1+|n|)^{\alpha-q},
$$

where the operator $\omega$ is defined for all $(t, n) \in \mathbb{T} \times \mathbb{Z}$ by $(\omega h)(t, n):=h(t, n+1)-$ $h(t, n)$.

$\alpha$ is called the order of the pseudo-differential operator $H$. The set of pseudo-differential operators of order $\alpha$ on $\mathbb{T}$ is denoted $\Psi^{\alpha}$ and we define

$$
\Psi^{-\infty}:=\bigcap_{\alpha \in \mathbb{R}} \Psi^{\alpha}
$$

Example 8. For further use, we introduce the example of multiplication operators. Consider $H: C^{\infty}(\mathbb{T}) \rightarrow C^{\infty}(\mathbb{T})$ defined as

$$
(H f)(t):=h(t) f(t), \quad h \in C^{\infty}(\mathbb{T}) .
$$

Decomposing in Fourier series, one immediately obtains

$$
(H f)=\sum_{n \in \mathbb{Z}} h \widehat{f}(n) e_{n} .
$$

There holds $\omega^{q} h=0$ for all $q \geq 1$ and, as $h \in C^{\infty}(\mathbb{T})$, for all $t \in \mathbb{T}$ we obtain

$$
\left|\left(\frac{d^{p} h}{d t^{p}}\right)(t)\right| \leq c_{p}, \quad \text { for some } c_{p}>0 .
$$

We get $H \in \Psi^{0}$.

Using the map $U$ defined in (8), we define periodic pseudo-differential operators on $\partial \Omega$ as follows.

\begin{tabular}{|l|l|l|l|l|l|}
\hline $\mathbf{2} 2 \mathbf{2 0}$ & $\mathbf{3 9 5}$ & $\mathbf{B}$ & $\begin{array}{l}\text { Dispatch: 30/1/2021 } \\
\text { Total pages: 38 } \\
\text { Disk Received } \\
\text { Disk Used } \square\end{array}$ & $\begin{array}{l}\text { Journal: Commun. Math. Phys. } \\
\text { Not Used } \square \\
\text { Corrupted } \\
\text { Mismatch } \square\end{array}$ \\
\hline
\end{tabular}


Definition 9. A linear operator $H$ on $C^{\infty}(\partial \Omega)$ is a periodic pseudo-differential operator on $\partial \Omega$ of order $\alpha \in \mathbb{R}$ if the operator $H_{0}:=U H U^{-1} \in \Psi^{\alpha}$. The set of pseudo differential operators on $\partial \Omega$ of order $\alpha$ is denoted $\Psi_{\partial \Omega}^{\alpha}$ and we set

$$
\Psi_{\partial \Omega}^{-\infty}:=\bigcap_{\alpha \in \mathbb{R}} \Psi_{\partial \Omega}^{\alpha}
$$

We will need the following properties of pseudo-differential operators on $\partial \Omega$. They can be found in [34, §5.8 \& 5.9].

Proposition 10. Let $s, \alpha, \beta \in \mathbb{R}$ and $H \in \Psi_{\partial \Omega}^{\alpha}, G \in \Psi_{\partial \Omega}^{\beta}$.

1. $H$ extends uniquely to a bounded linear operator, also denoted $H$, from $H^{s}(\partial \Omega)$ to $H^{s-\alpha}(\partial \Omega)$.

2. There holds

$$
H+G \in \Psi_{\partial \Omega}^{\max (\alpha, \beta)}, \quad H G \in \Psi_{\partial \Omega}^{\alpha+\beta}, \quad[H, G] \in \Psi_{\partial \Omega}^{\alpha+\beta-1} .
$$

2.3. Cauchy singular integral operators. For $f \in C^{\infty}(\partial \Omega)$, the Cauchy singular integral operator is defined as a principal value by

$$
S_{\mathrm{h}}(f)(z):=\frac{1}{\mathrm{i} \pi} \mathrm{p} \cdot \mathrm{v} \cdot \int_{\partial \Omega} \frac{f(\xi)}{\xi-z} d \xi, \quad z \in \partial \Omega .
$$

We define its anti-holomorphic counterpart as

$$
S_{\mathrm{ah}}(f)(z):=\overline{S_{\mathrm{h}}(\bar{f})(z)}=-\frac{1}{\mathrm{i} \pi} \mathrm{p} \cdot \mathrm{v} \cdot \int_{\partial \Omega} \frac{f(\xi)}{\overline{\bar{\xi}}-\bar{z}} d \bar{\xi}, \quad z \in \partial \Omega .
$$

It turns out $S_{\mathrm{h}}$ and $S_{\mathrm{ah}}$ are periodic pseudo-differential operators on $\partial \Omega$. This is the purpose of the following proposition.

Proposition 11. The linear maps $S_{\mathrm{h}}$ and $S_{\mathrm{ah}}$ are periodic pseudo-differential operators of order 0 . In particular, they are bounded linear operators from $H^{S}(\partial \Omega)$ onto itself for all $s \in \mathbb{R}$.

Proof. This is proved in [9, Prop. 2.9.] where the operators $S_{\mathrm{h}}$ and $S_{\text {ah }}$ are denoted $C_{\Sigma}$ and $-C_{\Sigma}^{\prime}$ respectively (with $\Sigma:=\partial \Omega$ ).

We will also need the following property.

Proposition 12. Let $H_{\mathbf{n}}$ be the multiplication operator by the normal $\mathbf{n}$ in $C^{\infty}(\partial \Omega)$. There holds:

1. $H_{\mathbf{n}}$ is a periodic pseudo-differential operator of order 0.

2. Let $\sharp \in\{\mathrm{h}$, ah $\}$ we have $\left[H_{\mathbf{n}}, S_{\sharp}\right] \in \Psi_{\partial \Omega}^{-1}$.

3. There holds $S_{\mathrm{ah}}+S_{\mathrm{h}} \in \Psi_{\partial \Omega}^{-\infty}$.

\begin{tabular}{|l|l|l|l|l|l|}
\hline $\mathbf{2} 2 \mathbf{2 0}$ & $\mathbf{3 9 5 9}$ & $\mathbf{B}$ & $\begin{array}{l}\text { Dispatch: 30/1/2021 } \\
\text { Total pages: 38 } \\
\text { Disk Received } \\
\text { Disk Used } \square\end{array}$ & $\begin{array}{l}\text { Journal: Commun. Math. Phys. } \\
\text { Not Used } \square \\
\text { Corrupted } \\
\text { Mismatch }\end{array}$ \\
\hline Jour. No
\end{tabular}


Proof. Point (1) is proved remarking that the operator $U H_{\mathbf{n}} U^{-1}$ is a multiplication operator in $\mathbb{T}$. Thanks to Example 8, we know that $U H_{\mathbf{n}} U^{-1} \in \Psi^{0}$ hence by definition we get $H_{\mathbf{n}} \in \Psi_{\partial \Omega}^{0}$.

Let us deal with Point (2). Let $\sharp \in\left\{\mathrm{h}\right.$, ah\}, by Proposition 11, $S_{\sharp} \in \Psi_{\partial \Omega}^{0}$ and by Point (1) $H_{\mathbf{n}} \in \Psi_{\partial \Omega}^{0}$. Hence, by (2) Proposition 10, we obtain Point (2).

Finally, we prove Point (3). By [9, Proposition 2.9.] there exists $L \in \Psi_{\partial \Omega}^{0}$ and $R_{1}, R_{2} \in \Psi_{\partial \Omega}^{-\infty}$ such that

$$
S_{\mathrm{h}}=L+R_{1}, \quad S_{\mathrm{ah}}=-L+R_{2} .
$$

Hence, $S_{\mathrm{h}}+S_{\mathrm{ah}}=R_{1}+R_{2} \in \Psi_{\partial \Omega}^{-\infty}$ by (2) Proposition 10 .

\section{Maximal Wirtinger Operators}

In this section we describe elementary properties of the maximal Wirtinger operators defined as

$$
\begin{aligned}
\partial_{\mathrm{h}} u & =\partial_{\bar{z}} u, \quad \operatorname{dom}\left(\partial_{\mathrm{h}}\right):=\left\{u \in L^{2}(\Omega): \partial_{\bar{z}} u \in L^{2}(\Omega)\right\}, \\
\partial_{\mathrm{ah}} u & =\partial_{z} u, \quad \operatorname{dom}\left(\partial_{\mathrm{ah}}\right):=\left\{u \in L^{2}(\Omega): \partial_{z} u \in L^{2}(\Omega)\right\} .
\end{aligned}
$$

For $\sharp \in\{h$, ah $\}$, consider the operator norms $\|\cdot\|_{\sharp}$ defined as

$$
\|u\|_{\sharp}^{2}:=\left\|\partial_{\sharp} u\right\|_{L^{2}(\Omega)}^{2}+\|u\|_{L^{2}(\Omega)}^{2}, \quad u \in \operatorname{dom}\left(\partial_{\sharp}\right) .
$$

In particular, $\operatorname{dom}\left(\partial_{\sharp}\right)$ endowed with the scalar product defined for $u, v \in \operatorname{dom}\left(\partial_{\sharp}\right)$ by

$$
\langle u, v\rangle_{\sharp}=\left\langle\partial_{\sharp} u, \partial_{\sharp} v\right\rangle_{L^{2}(\Omega)}+\langle u, v\rangle_{L^{2}(\Omega)},
$$

is a Hilbert space.

The first lemma is obtained by a simple integration by parts.

Lemma 13. The following identities hold.

$$
H^{1}\left(\mathbb{R}^{2}\right)=\left\{f \in L^{2}\left(\mathbb{R}^{2}\right): \partial_{z} f \in L^{2}\left(\mathbb{R}^{2}\right)\right\}=\left\{f \in L^{2}\left(\mathbb{R}^{2}\right): \partial_{\bar{z}} f \in L^{2}\left(\mathbb{R}^{2}\right)\right\} .
$$

Proof. Let $f \in C_{0}^{\infty}\left(\mathbb{R}^{2}\right)$. Integrating by parts several times we obtain:

$$
\begin{aligned}
\|\nabla f\|_{L^{2}\left(\mathbb{R}^{2} ; \mathbb{C}^{2}\right)}^{2}=\langle f,-\Delta f\rangle_{L^{2}\left(\mathbb{R}^{2}\right)} & =4\left\langle f,-\partial_{z} \partial_{\bar{z}} f\right\rangle_{L^{2}\left(\mathbb{R}^{2}\right)}=4\left\|\partial_{\bar{z}} f\right\|_{L^{2}\left(\mathbb{R}^{2}\right)}^{2} \\
& =4\left\langle f,-\partial_{\bar{z}} \partial_{z} f\right\rangle_{L^{2}\left(\mathbb{R}^{2}\right)}=4\left\|\partial_{z} f\right\|_{L^{2}\left(\mathbb{R}^{2}\right)}^{2}
\end{aligned}
$$

As $C_{0}^{\infty}\left(\mathbb{R}^{2}\right)$ is dense in $H^{1}\left(\mathbb{R}^{2}\right)$, we obtain the expected result.

The next lemma is a density result.

Lemma 14. Let $\sharp \in\{\mathrm{h}$, ah $\}$. The space $C^{\infty}(\bar{\Omega}):=C^{\infty}(\bar{\Omega}, \mathbb{C})$ is dense in $\operatorname{dom}\left(\partial_{\sharp}\right)$.

\begin{tabular}{|l|l|l|l|l|l|}
\hline $\mathbf{2} 2 \mathbf{2 0}$ & $\mathbf{3 9 5 9}$ & $\mathbf{B}$ & $\begin{array}{l}\text { Dispatch: 30/1/2021 } \\
\text { Total pages: 38 } \\
\text { Disk Received } \\
\text { Disk Used } \square\end{array}$ & $\begin{array}{l}\text { Journal: Commun. Math. Phys. } \\
\text { Not Used } \square \\
\text { Corrupted } \square \\
\text { Mismatch } \square\end{array}$ \\
\hline
\end{tabular}


Proof. Let $u \in \operatorname{dom}\left(\partial_{\mathrm{h}}\right)$ and assume that for all $\varphi \in C^{\infty}(\bar{\Omega})$ there holds

$$
0=\langle u, \varphi\rangle_{\mathrm{h}}=\left\langle\partial_{\bar{z}} u, \partial_{\bar{z}} \varphi\right\rangle_{L^{2}(\Omega)}+\langle u, \varphi\rangle_{L^{2}(\Omega)} .
$$

In particular, if $\varphi \in C_{0}^{\infty}(\Omega)$, we obtain $-\Delta u=-4 u$ first in $\mathcal{D}(\Omega)^{\prime}$ then in $L^{2}(\Omega)$. Define $v=\partial_{\bar{z}} u$ and denote by $v_{0}$ its extension to the whole $\mathbb{R}^{2}$ by 0 . For $\varphi \in C_{0}^{\infty}\left(\mathbb{R}^{2}\right)$ there holds

$$
\begin{aligned}
\left\langle\partial_{z} v_{0}, \bar{\varphi}\right\rangle_{\mathcal{D}^{\prime}\left(\mathbb{R}^{2}\right), \mathcal{D}\left(\mathbb{R}^{2}\right)} & =-\left\langle v_{0}, \overline{\partial_{\bar{z}} \varphi}\right\rangle_{\mathcal{D}^{\prime}\left(\mathbb{R}^{2}\right), \mathcal{D}\left(\mathbb{R}^{2}\right)} \\
& =-\left\langle v, \partial_{\bar{z}} \varphi\right\rangle_{L^{2}(\Omega)} \\
& =-\left\langle\partial_{\bar{z}} u, \partial_{\bar{z}} \varphi\right\rangle_{L^{2}(\Omega)} \\
& =\langle u, \varphi\rangle_{L^{2}(\Omega)} \\
& =\left\langle u_{0}, \varphi\right\rangle_{L^{2}\left(\mathbb{R}^{2}\right)} \\
& =\left\langle u_{0}, \bar{\varphi}\right\rangle_{\mathcal{D}^{\prime}\left(\mathbb{R}^{2}\right), \mathcal{D}\left(\mathbb{R}^{2}\right)}
\end{aligned}
$$

where $u_{0}$ denotes the extension by zero of $u$ to the whole $\mathbb{R}^{2}$. It gives $\partial_{z} v_{0}=u_{0} \in$ $L^{2}\left(\mathbb{R}^{2}\right)$. By Lemma $13, v_{0}$ is in $H^{1}\left(\mathbb{R}^{2}\right)$ and by [14, Prop. IX.18.] we get $v \in H_{0}^{1}(\Omega)$. Remark that in $\mathcal{D}^{\prime}(\Omega)$, there holds $\partial_{\bar{z}} \partial_{z} v=v$. Indeed, we have

$$
\partial_{\bar{z}} \partial_{z} v=\partial_{\bar{z}} \partial_{z} \partial_{\bar{z}} u=\partial_{\bar{z}} u=v .
$$

In particular this identity also holds true in $L^{2}(\Omega)$. Now, pick a sequence $v_{n} \in C_{0}^{\infty}(\Omega)$ converging to $v$ in the $H^{1}(\Omega)$-norm. There holds

$$
\begin{aligned}
\left\langle v, v_{n}\right\rangle_{L^{2}(\Omega)}=\left\langle\partial_{z} \partial_{\bar{z}} v, v_{n}\right\rangle_{L^{2}(\Omega)} & =-\left\langle\partial_{\bar{z}} v, \overline{\partial_{\bar{z}} v_{n}}\right\rangle_{\mathcal{D}^{\prime}(\Omega), \mathcal{D}(\Omega)} \\
& =-\left\langle\partial_{\bar{z}} v, \partial_{\bar{z}} v_{n}\right\rangle_{L^{2}(\Omega)}
\end{aligned}
$$

Letting $n \rightarrow+\infty$ one obtains $\|v\|_{L^{2}(\Omega)}^{2}=-\left\|\partial_{\bar{z}} v\right\|_{L^{2}(\Omega)}^{2}$ which implies $v=0$. In $\mathcal{D}^{\prime}(\Omega)$ we have $\partial_{z} v=\partial_{z} \partial_{\bar{z}} u=u$. As $v=0, u=0$ which concludes the proof for $\sharp=\mathrm{h}$. The case $\sharp=$ ah is handled similarly.

In order to describe precisely the domains $\operatorname{dom}\left(\partial_{\sharp}\right)(\sharp \in\{h, a h\})$ we need to prove the existence of traces on $\partial \Omega$ for functions in $\operatorname{dom}\left(\partial_{\sharp}\right)$. To this aim, define the following Dirichlet trace operators

$$
\Gamma^{+}: H^{1}(\Omega) \rightarrow H^{\frac{1}{2}}(\partial \Omega), \quad \Gamma^{-}: H_{l o c}^{1}\left(\mathbb{R}^{2} \backslash \Omega\right) \rightarrow H^{\frac{1}{2}}(\partial \Omega),
$$

where $H_{l o c}^{1}\left(\mathbb{R}^{2} \backslash \Omega\right)$ is the Fréchet space of locally $H^{1}$-functions in $\mathbb{R}^{2} \backslash \Omega$. These linear operators are known to be continuous (see [29, Thm. 3.37]). Moreover, there exists continuous extension operators such that for $f \in H^{\frac{1}{2}}(\partial \Omega)$ there holds

$$
E^{+} f \in H^{1}(\Omega), \quad E^{-} f \in H^{1}\left(\mathbb{R}^{2} \backslash \bar{\Omega}\right) \text { and } \Gamma^{ \pm} E^{ \pm} f=f .
$$

Actually, the operator $\Gamma^{+}$can be extended to functions in $\operatorname{dom}\left(\partial_{\sharp}\right)(\sharp \in\{h, a h\})$. This is the purpose of the following proposition.

Lemma 15. Let $\sharp \in\{\mathrm{h}, \mathrm{ah}\}$. The operator $\Gamma^{+}$defined in (9) extends into a linear bounded operator between $\operatorname{dom}\left(\partial_{\sharp}\right)$ and $H^{-\frac{1}{2}}(\partial \Omega)$.

\begin{tabular}{|l|l|l|l|l|l|}
\hline $\mathbf{2} 2 \mathbf{2 0}$ & $\mathbf{3 9 5 9}$ & $\mathbf{B}$ & $\begin{array}{l}\text { Dispatch: 30/1/2021 } \\
\text { Total pages: 38 } \\
\text { Disk Received } \\
\text { Disk Used } \square\end{array}$ & $\begin{array}{l}\text { Journal: Commun. Math. Phys. } \\
\text { Not Used } \square \\
\text { Corrupted } \square \\
\text { Mismatch } \square\end{array}$ \\
\hline Jour. No & Ms. No.
\end{tabular}


Proof. Let $\left(v_{n}\right)_{n \in \mathbb{N}} \in C^{\infty}(\bar{\Omega})^{\mathbb{N}}$ be a sequence that converges to $v$ in the $\|\cdot\|_{\text {h }}$-norm when $n \rightarrow+\infty$. Let us prove that $\left(\Gamma^{+} v_{n}\right)_{n \in \mathbb{N}}$ has a limit in $H^{-\frac{1}{2}}(\partial \Omega)$. First recall the integration by part formula

$$
\frac{1}{2}\left\langle\Gamma^{+} u, \overline{\mathbf{n}} \Gamma^{+} w\right\rangle_{L^{2}(\partial \Omega)}=\left\langle\partial_{\bar{z}} u, w\right\rangle_{L^{2}(\Omega)}+\left\langle u, \partial_{z} w\right\rangle_{L^{2}(\Omega)},
$$

valid for any $u, w \in H^{1}(\Omega)$. Second, pick $f \in H^{\frac{1}{2}}(\partial \Omega)$ and consider $w=E^{+}(\mathbf{n} f) \in$ $H^{1}(\Omega)$. There holds

$$
\left\langle\Gamma^{+}\left(v_{n}-v_{m}\right), f\right\rangle_{L^{2}(\partial \Omega)}=2\left\langle\partial_{\bar{z}}\left(v_{n}-v_{m}\right), w\right\rangle_{L^{2}(\Omega)}+2\left\langle v_{n}-v_{m}, \partial_{z} w\right\rangle_{L^{2}(\Omega)} .
$$

In particular, we have

$$
\begin{aligned}
\left|\left\langle\Gamma^{+}\left(v_{n}-v_{m}\right), f\right\rangle_{L^{2}(\partial \Omega)}\right| \leq & 2\left\|\partial_{\bar{z}}\left(v_{n}-v_{m}\right)\right\|_{L^{2}(\Omega)}\|w\|_{L^{2}(\Omega)} \\
& +2\left\|v_{n}-v_{m}\right\|_{L^{2}(\Omega)}\left\|\partial_{z} w\right\|_{L^{2}(\Omega)} \\
\leq & 4\|w\|_{H^{1}(\Omega)}\left\|v_{n}-v_{m}\right\|_{\mathrm{h}} \\
\leq & 4 c_{\Omega}\|f\|_{H^{\frac{1}{2}(\partial \Omega)}}\left\|v_{n}-v_{m}\right\|_{\mathrm{h}} \quad\left(\text { for some } c_{\Omega}>0\right),
\end{aligned}
$$

where we have used that $E^{+}$is a continuous linear map and that the multiplication operator by $\mathbf{n}$ is bounded from $H^{\frac{1}{2}}(\partial \Omega)$ onto itself. When $n, m \rightarrow+\infty$ we obtain $\left\|\Gamma^{+}\left(v_{n}-v_{m}\right)\right\|_{H^{-\frac{1}{2}}(\partial \Omega)} \rightarrow 0$. In particular $\left(\Gamma^{+} v_{n}\right)_{n \in \mathbb{N}}$ is a Cauchy sequence in $H^{-\frac{1}{2}}(\partial \Omega)$ thus converges to an element $g \in H^{-\frac{1}{2}}(\partial \Omega)$ and we define $\Gamma^{+} v:=g$. Remark that the definition of $\Gamma^{+} v$ does not depend on the chosen sequence $\left(v_{n}\right)_{n \in \mathbb{N}}$ and that we have

$$
\left\|\Gamma_{+} v_{n}\right\|_{H^{-\frac{1}{2}(\partial \Omega)}} \leq 4 c_{\Omega}\left\|v_{n}\right\|_{h}
$$

which implies, when $n \rightarrow+\infty$, that $\Gamma^{+}$is bounded from $\operatorname{dom}\left(\partial_{\mathrm{h}}\right)$ to $H^{-\frac{1}{2}}(\partial \Omega)$. The proof for dom $\left(\partial_{\mathrm{ah}}\right)$ is handled similarly.

Remark 16. If one picks $R>0$ such that $\bar{\Omega} \subset B(0, R):=\left\{x \in \mathbb{R}^{2}:\|x\|<R\right\}$, one can prove that for $\star \in\{z, \bar{z}\}, \Gamma^{-}$extends into a linear bounded operator between the space $\left\{u \in L^{2}(B(0, R) \backslash \Omega): \partial_{\star} u \in L^{2}(B(0, R) \backslash \Omega)\right\}$ and $H^{-\frac{1}{2}}(\partial \Omega)$. The proof goes along the same lines as the one of Lemma 15 , using an extension operator $E^{-}: H^{\frac{1}{2}}(\partial \Omega) \rightarrow$ $H^{1}(B(0, R) \backslash \Omega)$ constructed such that for all $f \in H^{\frac{1}{2}}(\partial \Omega),\left.E^{-}(f)\right|_{\partial B(0, R)}=0$.

Remark 17. Pick $u \in \operatorname{dom}\left(\partial_{\mathrm{ah}}\right)$ and $w \in H^{1}(\Omega)$. Note that by definition, the following Green's Formula holds

$$
\left\langle\partial_{z} u, w\right\rangle_{L^{2}(\Omega)}=-\left\langle u, \partial_{\bar{z}} w\right\rangle_{L^{2}(\Omega)}+\frac{1}{2}\left\langle\overline{\mathbf{n}} \Gamma^{+} u, \Gamma^{+} w\right\rangle_{H^{-\frac{1}{2}}(\partial \Omega), H^{\frac{1}{2}}(\partial \Omega)} .
$$

The following elliptic regularity result is rather well known (see the analogous statement [11, Lemma 2.4.]).

Lemma 18. Let $\sharp \in\{\mathrm{h}$, ah $\}$ and $u \in \operatorname{dom}\left(\partial_{\sharp}\right)$. If $\Gamma^{+} u \in H^{\frac{1}{2}}(\partial \Omega)$ then $u \in H^{1}(\Omega)$. 
Proof. Let $u \in \operatorname{dom}\left(\partial_{\mathrm{h}}\right)$ be such that $\Gamma^{+} u \in H^{\frac{1}{2}}(\partial \Omega)$ and set $v=u-E^{+}\left(\Gamma^{+} u\right)$. Then, $\Gamma^{+} v=0$ and if $v \in H_{0}^{1}(\Omega)$ the result is proved. If $v_{n} \in C^{\infty}(\bar{\Omega})$ is a sequence converging to $v$ in the $\|\cdot\|_{\mathrm{h}}$-norm there holds $\Gamma^{+} v_{n} \rightarrow 0$ in $H^{-\frac{1}{2}}(\partial \Omega)$ by Lemma 15 . In particular, it gives for any $w \in H^{1}(\Omega)$

$$
\begin{aligned}
\left\langle v, \partial_{z} w\right\rangle_{L^{2}(\Omega)} & =\lim _{n \rightarrow+\infty}\left(-\left\langle\partial_{\bar{z}} v_{n}, w\right\rangle_{L^{2}(\Omega)}+\frac{1}{2}\left\langle\Gamma^{+} v_{n}, \overline{\mathbf{n}} \Gamma^{+} w\right\rangle_{L^{2}(\partial \Omega)}\right) \\
& =-\left\langle\partial_{\bar{z}} v, w\right\rangle_{L^{2}(\Omega)} .
\end{aligned}
$$

Let $v_{0}$ (resp. $h_{0}$ ) be the extension of $v$ (resp. $h:=\partial_{\bar{z}} v$ ) by zero to the whole $\mathbb{R}^{2}$. If $\varphi \in C_{0}^{\infty}\left(\mathbb{R}^{2}\right)$, there holds

$$
\begin{aligned}
-\left\langle h_{0}, \bar{\varphi}\right\rangle_{\mathcal{D}^{\prime}\left(\mathbb{R}^{2}\right), \mathcal{D}\left(\mathbb{R}^{2}\right)} & =-\langle h, \varphi\rangle_{L^{2}(\Omega)}=\left\langle v, \partial_{z} \varphi\right\rangle_{L^{2}(\Omega)} \\
& =\left\langle v_{0}, \partial_{\bar{z}} \bar{\varphi}\right\rangle_{\mathcal{D}^{\prime}\left(\mathbb{R}^{2}\right), \mathcal{D}\left(\mathbb{R}^{2}\right)} \\
& =-\left\langle\partial_{\bar{z}} v_{0}, \bar{\varphi}\right\rangle_{\mathcal{D}^{\prime}\left(\mathbb{R}^{2}\right), \mathcal{D}\left(\mathbb{R}^{2}\right)} .
\end{aligned}
$$

Thus $\partial_{\bar{z}} v_{0}=h_{0} \in L^{2}\left(\mathbb{R}^{2}\right)$ and by Lemma $13, v_{0} \in H^{1}\left(\mathbb{R}^{2}\right)$ and $v \in H_{0}^{1}(\Omega)$. The proof for $u \in \operatorname{dom}\left(\partial_{\mathrm{ah}}\right)$ is handled similarly.

\section{Bergman and Hardy Spaces on $\boldsymbol{\Omega}$}

We introduce $\mathcal{A}_{\mathrm{h}}^{2}(\Omega)$ and $\mathcal{A}_{\mathrm{ah}}^{2}(\Omega)$ the holomorphic and anti-holomorphic Bergman spaces on $\Omega$, respectively. They are defined as

$$
\mathcal{A}_{\mathrm{h}}^{2}(\Omega):=\left\{u \in \operatorname{Hol}(\Omega) \cap L^{2}(\Omega)\right\}, \quad \mathcal{A}_{\mathrm{ah}}^{2}(\Omega):=\left\{u: \bar{u} \in \mathcal{A}_{\mathrm{h}}^{2}(\Omega)\right\},
$$

where $\operatorname{Hol}(\Omega)$ denotes the space of holomorphic functions in $\Omega$. The holomorphic and anti-holomorphic Hardy spaces, denoted $\mathcal{H}_{\mathrm{h}}^{2}(\Omega)$ and $\mathcal{H}_{\text {ah }}^{2}(\Omega)$, respectively, are defined as

$$
\mathcal{H}_{\mathrm{h}}^{2}(\Omega):=\left\{u \in \mathcal{A}_{\mathrm{h}}^{2}(\Omega): \Gamma^{+} u \in L^{2}(\partial \Omega)\right\}, \quad \mathcal{H}_{\mathrm{ah}}^{2}(\Omega):=\left\{u: \bar{u} \in \mathcal{H}_{\mathrm{h}}^{2}(\Omega)\right\} .
$$

This section aims to describe explicitely the Bergman and Hardy spaces on $\Omega$ in terms of Cauchy integrals and Szegó projectors that we define now.

For $f \in C^{\infty}(\partial \Omega)$ consider the Cauchy integrals defined for $z \in \mathbb{C} \backslash \partial \Omega$ by

$$
\Phi_{\mathrm{h}}(f)(z):=\frac{1}{2 \mathrm{i} \pi} \int_{\partial \Omega} \frac{f(\xi)}{\xi-z} d \xi, \quad \Phi_{\mathrm{ah}}(f)(z):=-\frac{1}{2 \mathrm{i} \pi} \int_{\partial \Omega} \frac{f(\xi)}{\bar{\xi}-\bar{z}} d \bar{\xi} .
$$

It is well known (see [34, §4.1.2.]) that $\Phi_{\mathrm{h}}(f)$ (resp. $\Phi_{\mathrm{ah}}(f)$ ) defines a holomorphic function (resp. anti-holomorphic function) in $\mathbb{R}^{2} \backslash \partial \Omega$.

The well-known Plemelj-Sokhotski formula (see [34, Thm. 4.1.1]) states that for $f \in C^{\infty}(\partial \Omega)$ the functions $\Phi_{\mathrm{h}}(f)$ and $\Phi_{\mathrm{ah}}(f)$ have an interior and an exterior Dirichlet trace, denoted respectively $\gamma_{0}^{+}$and $\gamma_{0}^{-}$, such that:

$$
\gamma_{0}^{ \pm} \Phi_{\mathrm{h}}(f)= \pm \frac{1}{2} f+\frac{1}{2} S_{\mathrm{h}} f, \quad \gamma_{0}^{ \pm} \Phi_{\mathrm{ah}}(f)= \pm \frac{1}{2} f+\frac{1}{2} S_{\mathrm{ah}} f .
$$

Let $\sharp \in\left\{\mathrm{h}\right.$, ah\}, note that by [10, Theorem 3.1.], for $f \in C^{\infty}(\partial \Omega)$ we know that $\left.\Phi_{\sharp}(f)\right|_{\Omega} \in C^{\infty}(\bar{\Omega})$ as well as $\left.\Phi_{\sharp}(f)\right|_{\mathbb{R}^{2} \backslash \bar{\Omega}} \in C^{\infty}\left(\mathbb{R}^{2} \backslash \Omega\right)$. In particular, the traces $\gamma_{0}^{ \pm} \Phi_{\sharp}(f)$ coincide with $\Gamma^{ \pm} \Phi_{\sharp}(f)$, where $\Gamma^{ \pm}$are the trace operators defined in Lemma 15 and Remark 16.

\begin{tabular}{|l|l|l|l|l|l|}
\hline 220 & $\mathbf{3 9 5 9} 2$ & $\mathbf{B}$ & $\begin{array}{l}\text { Dispatch: 30/1/2021 } \\
\text { Total pages: 38 } \\
\text { Disk Received } \\
\text { Disk Used } \square\end{array}$ & $\begin{array}{l}\text { Journal: Commun. Math. Phys. } \\
\text { Not Used } \\
\text { Corrupted } \\
\text { Mismatch } \square\end{array}$ \\
\hline Jour. No & Ms. No.
\end{tabular}


Definition 19. We define the Szegő projectors in $C^{\infty}(\partial \Omega)$ by

$$
\Pi_{\mathrm{h}}^{ \pm}:= \pm \Gamma^{ \pm} \Phi_{\mathrm{h}}, \quad \Pi_{\mathrm{ah}}^{ \pm}:= \pm \Gamma^{ \pm} \Phi_{\mathrm{ah}} .
$$

Proposition 20. Let $s \in \mathbb{R}$ and $\sharp \in\left\{\mathrm{h}\right.$, ah\}. The Szegó projectors $\Pi_{\sharp}^{ \pm}$extend uniquely into bounded linear operators from $H^{s}(\partial \Omega)$ onto itself. Moreover, $\Pi_{\sharp}^{ \pm}$are projectors and $\Pi_{\sharp}^{+}+\Pi_{\sharp}^{-}=1$.

Proof. Remark that for $\sharp \in\{\mathrm{h}, \mathrm{ah}\}$ and $f \in C^{\infty}(\partial \Omega)$, there holds

$$
\Pi_{\sharp}^{ \pm} f=\frac{1}{2} f \pm \frac{1}{2} S_{\sharp} f .
$$

By Proposition 11, $\Pi_{\sharp}^{ \pm}$extends into a bounded linear operator from $H^{s}(\partial \Omega)$ onto itself for all $s \in \mathbb{R}$.

Let $s \in \mathbb{R}$ and $f \in H^{s}(\partial \Omega)$. A fundamental fact is that $S_{\mathrm{h}}^{2} f=f$ (see [34, Eq. (4.10)]), in particular it implies that $S_{\text {ah }}^{2} f=f$. Hence, we obtain

$$
\begin{aligned}
\left(\Pi_{\sharp}^{ \pm}\right)^{2} & =\left(\frac{1}{2} \pm \frac{1}{2} S_{\sharp}\right)\left(\frac{1}{2} \pm \frac{1}{2} S_{\sharp}\right) \\
& =\frac{1}{4}+\frac{1}{4} S_{\sharp}^{2} \pm \frac{1}{2} S_{\sharp} \\
& =\frac{1}{2} \pm \frac{1}{2} S_{\sharp} \\
& =\Pi_{\sharp}^{ \pm} .
\end{aligned}
$$

Thus $\Pi_{\sharp}^{ \pm}$are projectors and one easily checks that $\Pi_{\sharp}^{+}+\Pi_{\sharp}^{-}=1$.

The main goal of this section is to prove the following description of the Bergman and Hardy spaces. As we will see further on in Proposition 22, this description relies on an extension of the operators $\Phi_{\sharp}$ to Sobolev spaces on the boundary $\partial \Omega(\sharp \in\{h, a h\})$.

Theorem 21. Let $\sharp \in\{\mathrm{h}, \mathrm{ah}\}$. The Bergman spaces satisfy

$$
\mathcal{A}_{\sharp}^{2}(\Omega)=\left\{\Phi_{\sharp}(f): f \in H^{-\frac{1}{2}}(\partial \Omega), \Pi_{\sharp}^{-} f=0\right\} .
$$

The Hardy spaces are given by

$$
\mathcal{H}_{\sharp}^{2}(\Omega)=\left\{\Phi_{\sharp}(f): f \in L^{2}(\partial \Omega), \Pi_{\sharp}^{-} f=0\right\} .
$$

4.1. Potential theory of the Wirtinger derivatives. In this paragraph we prove the following proposition.

Proposition 22. Let $\sharp \in\{\mathrm{h}, \mathrm{ah}\}$ and $s \in\left\{-\frac{1}{2}, 0, \frac{1}{2}\right\}$. The operator $\Phi_{\sharp}$ extends uniquely into a bounded operator from $H^{s}(\partial \Omega)$ to $H^{s+\frac{1}{2}}(\Omega)$ also denoted $\Phi_{\sharp}$.

In order to prove Proposition 22, we will need a few lemmas. Let us start by defining fundamental solutions of the Wirtinger operators $\partial_{\mathrm{h}}$ and $\partial_{\mathrm{ah}}$ :

$$
\varphi_{\mathrm{h}}(x)=\frac{1}{\pi\left(x_{1}+\mathrm{i} x_{2}\right)}, \quad \varphi_{\mathrm{ah}}(x)=\frac{1}{\pi\left(x_{1}-\mathrm{i} x_{2}\right)} .
$$

\begin{tabular}{|l|l|l|l|l|l|}
\hline $\mathbf{2} 2 \mathbf{2 0}$ & $\mathbf{3 9 5 9}$ & $\mathbf{B}$ & $\begin{array}{l}\text { Dispatch: 30/1/2021 } \\
\text { Total pages: 38 } \\
\text { Disk Received } \\
\text { Disk Used } \square\end{array}$ & $\begin{array}{l}\text { Journal: Commun. Math. Phys. } \\
\text { Not Used } \square \\
\text { Corrupted } \square \\
\text { Mismatch } \square\end{array}$ \\
\hline Jour. No & Ms. No.
\end{tabular}


Lemma 23. Let $\sharp \in\{\mathrm{h}$, ah\}. The linear map

$$
N_{\sharp}: u \in L^{2}(\Omega) \mapsto \varphi_{\sharp} * u_{0}
$$

is bounded from $L^{2}(\Omega)$ to $H^{1}(K)$ for all compact $K \subset \mathbb{R}^{2}$. Here $u_{0}$ denotes the extension of $u$ by zero to the whole $\mathbb{R}^{2}$.

Proof. Let us prove it for $\sharp=\mathrm{h}$ the proof for $\sharp=$ ah being similar. In the space of distributions $\mathcal{D}^{\prime}\left(\mathbb{R}^{2}\right)$, there holds

$$
\partial_{\bar{z}} \varphi_{\mathrm{h}}=\delta_{0},
$$

where $\delta_{0}$ is the Dirac delta-distribution.

Now, for $u$ in the Schwartz space $\mathcal{S}\left(\mathbb{R}^{2}\right)$ recall that the Fourier transform of $u$ is defined as

$$
\widehat{u}(k):=\int_{\mathbb{R}^{2}} u(x) e^{-2 \mathrm{i} \pi\langle x, k\rangle_{\mathbb{R}^{2}}} d x, \quad \text { for all } k \in \mathbb{R}^{2}
$$

and $\widehat{u} \in \mathcal{S}\left(\mathbb{R}^{2}\right)$. The Fourier transform extends to the space of tempered distribution $\mathcal{S}^{\prime}\left(\mathbb{R}^{2}\right)$ and as $\delta_{0} \in \mathcal{S}^{\prime}\left(\mathbb{R}^{2}\right)$, the Fourier transform of (14) yields

$$
\widehat{\varphi_{\mathrm{h}}}(k)=\frac{1}{\pi \mathrm{i}\left(k_{1}+\mathrm{i} k_{2}\right)}, \quad k=\left(k_{1}, k_{2}\right) \in \mathbb{R}^{2} \backslash\{(0,0)\} .
$$

Let $K$ be a compact subset of $\mathbb{R}^{2}$ and take $u \in L^{2}(\Omega)$. We extend $u$ by zero to $\mathbb{R}^{2}$ and denote this extension $u_{0} \in L^{2}\left(\mathbb{R}^{2}\right)$.

$$
\begin{aligned}
\left\|\varphi_{\mathrm{h}} * u_{0}\right\|_{H^{1}(K)}^{2} & \leq\left\|\varphi_{\mathrm{h}} * u_{0}\right\|_{L^{2}(K)}^{2}+\int_{\mathbb{R}^{2}}|k|^{2}\left|\left(\widehat{\varphi_{\mathrm{h}} * u_{0}}\right)(k)\right|^{2} d k \\
& =\left\|\varphi_{\mathrm{h}} * u_{0}\right\|_{L^{2}(K)}^{2}+\int_{\mathbb{R}^{2}}|k|^{2}\left|\widehat{\varphi_{\mathrm{h}}}(k) \widehat{u_{0}}(k)\right|^{2} d k \\
& =\left\|\varphi_{\mathrm{h}} * u_{0}\right\|_{L^{2}(K)}^{2}+\frac{1}{\pi^{2}} \int_{\mathbb{R}^{2}}\left|\widehat{u_{0}}(k)\right|^{2} d k \\
& =\left\|\varphi_{\mathrm{h}} * u_{0}\right\|_{L^{2}(K)}^{2}+\frac{1}{\pi^{2}}\|u\|_{L^{2}(\Omega)}^{2} .
\end{aligned}
$$

Now, let $R>0$ be such that $K \subset\left\{x \in \mathbb{R}^{2}:|x|<R\right\}$ and $\bar{\Omega} \subset\left\{x \in \mathbb{R}^{2}:|x|<R\right\}$. Consider a cut-off function $\chi \in C_{0}^{\infty}([0,+\infty)), 0 \leq \chi \leq 1$, such that

$$
\chi(\rho)=1 \text { whenever } 0 \leq \rho<2 R, \quad \chi(\rho)=0 \text { whenever } \rho>3 R .
$$

Define the function $u_{\chi}$ as

$$
u_{\chi}(x):=\int_{\mathbb{R}^{2}} \chi(|x-y|) \varphi_{\mathrm{h}}(x-y) u_{0}(y) d y .
$$

As defined, $\left.\left.u_{\chi}\right|_{K} \equiv\left(\varphi_{\mathrm{h}} * u_{0}\right)\right|_{K}$. Hence, we get

$$
\begin{aligned}
\left\|\varphi_{\mathrm{h}} * u_{0}\right\|_{L^{2}(K)}=\left\|u_{\chi}\right\|_{L^{2}(K)} & \leq\left\|u_{\chi}\right\|_{L^{2}\left(\mathbb{R}^{2}\right)} \\
& \leq\left\|\chi(|\cdot|) \varphi_{\mathrm{h}}\right\|_{L^{1}\left(\mathbb{R}^{2}\right)}\|u\|_{L^{2}(\Omega)},
\end{aligned}
$$


where we have used Young's inequality because $\chi(|\cdot|) \varphi_{\mathrm{h}} \in L^{1}\left(\mathbb{R}^{2}\right)$. Indeed, there holds

$$
\left\|\chi(|\cdot|) \varphi_{\mathrm{h}}\right\|_{L^{1}\left(\mathbb{R}^{2}\right)} \leq \frac{1}{\pi} \int_{B(0,3 R)} \frac{1}{|x|} d x=6 R .
$$

In particular, there exists $c_{K}>0$, such that

$$
\left\|\varphi_{\mathrm{h}} * u_{0}\right\|_{H^{1}(K)} \leq c_{K}\|u\|_{L^{2}(\Omega)} .
$$

Hence, for any compact $K \subset \mathbb{R}^{2}, N_{\sharp}$ is a bounded linear operator from $L^{2}(\Omega)$ to $H^{1}(K)$ and the proposition is proved.

Next, we recall that the Dirichlet trace on $\partial \Omega$ of a function in $H_{l o c}^{1}\left(\mathbb{R}^{2}\right)$ can be defined as

$$
\Gamma: H_{l o c}^{1}\left(\mathbb{R}^{2}\right) \rightarrow H^{\frac{1}{2}}(\partial \Omega)
$$

and is a continuous linear operator from $H_{l o c}^{1}\left(\mathbb{R}^{2}\right)$ to $H^{\frac{1}{2}}(\partial \Omega)$ (see [29, Thm. 3.37]).

Moreover, for $s \in[0, \ell]$, we introduce $\mathbf{t}(s):=\gamma_{1}^{\prime}(s)+\mathrm{i} \gamma_{2}^{\prime}(s)$ the expression of the tangent vector in the complex plane at the point $\gamma_{1}(s)+\mathrm{i} \gamma_{2}(s)$.

Lemma 24. The dual adjoints of $\left(\mathbf{t} \Gamma N_{\mathrm{h}}\right)$ and $\left(\overline{\mathbf{t}} \Gamma N_{\mathrm{ah}}\right)$, denoted $\left(\mathbf{t} \Gamma N_{\mathrm{h}}\right)^{\prime}$ and $\left(\overline{\mathbf{t}} \Gamma N_{\mathrm{ah}}\right)^{\prime}$ respectively, are bounded linear maps from $H^{-\frac{1}{2}}(\partial \Omega)$ to $L^{2}(\Omega)$. Moreover if $f \in$ $C^{\infty}(\partial \Omega)$, in $L^{2}(\Omega)$ there holds:

$$
\Phi_{\mathrm{ah}}(f)=\frac{\mathrm{i}}{2}\left(\mathbf{t} \Gamma N_{\mathrm{h}}\right)^{\prime}(f), \quad \Phi_{\mathrm{h}}(f)=-\frac{\mathrm{i}}{2}\left(\overline{\mathbf{t}} \Gamma N_{\mathrm{ah}}\right)^{\prime}(f) .
$$

Proof. Thanks to Lemma 23 and the mapping properties of $\Gamma$ we know that $\Gamma N_{\sharp}$ is a bounded linear map from $L^{2}(\Omega)$ to $H^{\frac{1}{2}}(\partial \Omega$ ) (for $\sharp \in\{\mathrm{h}$, ah $\}$ ). As $\Omega$ is smooth, $\mathbf{t} \in C^{\infty}(\partial \Omega)$ and $\overline{\mathbf{t}} \in C^{\infty}(\partial \Omega)$. In particular the multiplication operators by $\mathbf{t}$ and $\overline{\mathbf{t}}$ are bounded and invertible in $H^{\frac{1}{2}}(\Omega)$. Hence, their dual adjoints satisfy the expected mapping property.

Now, pick $f \in C^{\infty}(\partial \Omega)$ and $v \in L^{2}(\Omega)$. Denoting by $v_{0}$ the extension of $v$ by zero to the whole $\mathbb{R}^{2}$ and using Fubini's theorem, there holds

$$
\begin{aligned}
\left\langle\left(\mathbf{t} \Gamma N_{\mathrm{h}}\right)^{\prime} f, v\right\rangle_{L^{2}(\Omega)} & =\left\langle f, \mathbf{t} \Gamma N_{\mathrm{h}} v{ }_{H^{-\frac{1}{2}}(\partial \Omega), H^{\frac{1}{2}}(\partial \Omega)}\right. \\
& =\left\langle f, \mathbf{t} \Gamma N_{\mathrm{h}} v\right\rangle_{L^{2}(\partial \Omega)} \\
& =\int_{x \in \partial \Omega} \int_{y \in \mathbb{R}^{2}} \frac{f(x) \overline{v_{0}(y) \mathbf{t}(x)}}{\pi\left(\left(x_{1}-\mathrm{i} x_{2}\right)-\left(y_{1}-\mathrm{i} y_{2}\right)\right)} d y d s(x) \\
& =\int_{y \in \mathbb{R}^{2}} \frac{1}{\pi}\left(\int_{s=0}^{\ell} \frac{f(\gamma(s))\left(\gamma_{1}^{\prime}(s)-\mathrm{i} \gamma_{2}^{\prime}(s)\right)}{\left(\gamma_{1}(s)-\mathrm{i} \gamma_{2}(s)\right)-\left(y_{1}-\mathrm{i} y_{2}\right)} d s\right) \overline{v_{0}(y)} d y \\
& =\int_{y \in \mathbb{R}^{2}}\left(\frac{1}{\pi} \int_{\xi \in \partial \Omega} \frac{f(\xi)}{\bar{\xi}-\left(y_{1}-\mathrm{i} y_{2}\right)} d \bar{\xi}\right) \overline{v_{0}(y)} d y \\
& =\left\langle-2 \mathrm{i} \Phi_{\mathrm{ah}}(f), v\right\rangle_{L^{2}(\Omega)} .
\end{aligned}
$$

The proof for $\left(\overline{\mathbf{t}} \Gamma N_{\mathrm{ah}}\right)^{\prime}$ goes along the same lines, which concludes the proof of this lemma.

\begin{tabular}{|l|l|l|l|l|l|}
\hline $\mathbf{2} 2 \mathbf{2} 0$ & $\mathbf{3 9 5 9}$ & $\mathbf{B}$ & $\begin{array}{l}\text { Dispatch: 30/1/2021 } \\
\text { Total pages: } 38 \\
\text { Disk Received } \\
\text { Disk Used } \square\end{array}$
\end{tabular}$\quad$\begin{tabular}{l}
$\begin{array}{l}\text { Journal: Commun. Math. Phys. } \\
\text { Not Used } \\
\text { Corrupted } \\
\text { Mismatch }\end{array}$ \\
\hline Jour. No
\end{tabular}


For further use, we still denote $\Phi_{\mathrm{ah}}$ and $\Phi_{\mathrm{h}}$ the operators $\frac{\mathrm{i}}{2}\left(\mathbf{t} \Gamma N_{\mathrm{h}}\right)^{\prime}$ and $-\frac{\mathrm{i}}{2}\left(\overline{\mathbf{t}} \Gamma N_{\mathrm{ah}}\right)^{\prime}$. Now, for $\sharp \in\{h$, ah $\}$, when considering the operators

$$
\Phi_{\sharp}:\left(C^{\infty}(\partial \Omega),\|\cdot\|_{H^{-\frac{1}{2}}(\partial \Omega)}\right) \rightarrow\left(\operatorname{dom}\left(\partial_{\sharp}\right),\|\cdot\|_{\sharp}\right)
$$

they are bounded operators because for any $f \in C^{\infty}(\partial \Omega), \Phi_{\mathrm{h}}(f)$ and $\Phi_{\mathrm{ah}}(f)$ are holomorphic and anti-holomorphic in $\Omega$, respectively. The density of $C^{\infty}(\partial \Omega)$ in $H^{-\frac{1}{2}}(\partial \Omega)$ yields for each operator a unique extension to $H^{-\frac{1}{2}}(\partial \Omega)$ which coincides with the previous one. In particular, for any $f \in H^{-\frac{1}{2}}(\partial \Omega), \Phi_{\sharp}(f) \in \operatorname{dom}\left(\partial_{\sharp}\right)$ and $\partial_{\sharp} \Phi_{\sharp}(f)=0$.

Now, we have collected all the tools to prove Proposition 22.

Proof of Proposition 22. For $s=-\frac{1}{2}$, Proposition 22 holds true, because of Lemma 24 and the density of $C^{\infty}(\partial \Omega)$ in $H^{-\frac{1}{2}}(\partial \Omega)$. Let us prove it for $s=\frac{1}{2}$. Remark that $\Phi_{\sharp}(f) \in \operatorname{dom}\left(\partial_{\sharp}\right)$ so if $f \in H^{\frac{1}{2}}(\partial \Omega)$ we also have $\Gamma^{+} \Phi_{\sharp}(f)=\Pi_{\sharp}^{+} f \in H^{\frac{1}{2}}(\partial \Omega)$ by Proposition 20. Hence, by Lemma $18, \Phi_{\sharp}(f) \in H^{1}(\Omega)$.

Let us use the closed graph theorem and take a sequence of functions $f_{n} \in H^{\frac{1}{2}}(\partial \Omega)$ such that $f_{n} \rightarrow f$ in the $H^{\frac{1}{2}}(\partial \Omega)$-norm. Assume also that $\Phi_{\sharp}\left(f_{n}\right) \rightarrow u \in H^{1}(\Omega)$ where the convergence holds in the $H^{1}(\Omega)$-norm.

Because of the continuous embedding of $H^{\frac{1}{2}}(\partial \Omega)$ into $H^{-\frac{1}{2}}(\partial \Omega), f_{n} \rightarrow f$ also in the $H^{-\frac{1}{2}}(\partial \Omega)$-norm. In particular, by Proposition 22 for $s=-\frac{1}{2}, \Phi_{\sharp}\left(f_{n}\right) \rightarrow \Phi_{\sharp}(f)$ in $L^{2}(\Omega)$. Consequently, the equality $u=\Phi_{\sharp}(f)$ holds not only in $L^{2}(\Omega)$ but also in $H^{1}(\Omega)$ and by the closed graph theorem, $\Phi_{\sharp}$ is a continuous linear map between $H^{\frac{1}{2}}(\partial \Omega)$ and $H^{1}(\Omega)$.

The result for $s=0$ holds by (real) interpolation theory (see [35, Prop. 2.1.62. \& Prop. 2.3.11. \& Prop. 2.4.3.]).

4.2. Explicit description of the Bergman and Hardy spaces. Let us prove Theorem 21, starting with the following proposition concerning the Bergman spaces.

Proposition 25. Let $\sharp \in\{\mathrm{h}, \mathrm{ah}\}$. There holds:

$$
\mathcal{A}_{\sharp}^{2}(\Omega)=\left\{\Phi_{\sharp}(f): f \in H^{-\frac{1}{2}}(\partial \Omega) \text { such that } \Pi_{\sharp}^{-} f=0\right\}, \quad \sharp \in\{\mathrm{h}, \mathrm{ah}\} .
$$

Moreover, for all $f \in H^{-\frac{1}{2}}(\partial \Omega)$ there holds

$$
\Phi_{\sharp}(f)=\Phi_{\sharp}\left(\Pi_{\sharp}^{+} f\right) .
$$

\begin{tabular}{|l|l|l|l|l|l|}
\hline 220 & $\mathbf{3 9 5 9}$ & $\mathbf{B}$ & $\begin{array}{l}\text { Dispatch: 30/1/2021 } \\
\text { Total pages: 38 } \\
\text { Disk Received } \\
\text { Disk Used } \square\end{array}$ & $\begin{array}{l}\text { Journal: Commun. Math. Phys. } \\
\text { Not Used } \square \\
\text { Corrupted } \\
\text { Mismatch } \square\end{array}$ \\
\hline Jour. No & Ms. No.
\end{tabular}


Proof. Denote $\mathcal{E}_{\sharp}$ the set on the right-hand side of (15). We prove it for $\sharp=\mathrm{h}$, the proof for $\sharp=$ ah being similar.

Inclusion $\mathcal{E}_{\mathrm{h}} \subset \mathcal{A}_{\mathrm{h}}^{2}(\Omega)$ Let $u=\Phi_{\mathrm{h}}(f) \in \mathcal{E}_{\mathrm{h}}$, with $f \in H^{-\frac{1}{2}}(\partial \Omega)$ such that $\Pi_{\mathrm{h}}^{-} f=0$. By Proposition $22, \Phi_{\mathrm{h}}$ maps $H^{-\frac{1}{2}}(\partial \Omega)$ to $L^{2}(\Omega)$ thus $u \in L^{2}(\Omega)$. Moreover, there holds $\partial_{\bar{z}} u=0$ which implies that $u \in \mathcal{A}_{\mathrm{h}}^{2}(\Omega)$.

Inclusion $\mathcal{A}_{\mathrm{h}}^{2}(\Omega) \subset \mathcal{E}_{\mathrm{h}}$ For $u \in C^{\infty}(\bar{\Omega}), x \in \Omega$ and $\varepsilon>0$ sufficiently small there holds

$$
\begin{aligned}
0= & \frac{1}{\pi} \int_{\Omega \backslash B(x, \varepsilon)} \partial_{\bar{z}}\left(\frac{1}{\left(x_{1}+\mathrm{i} x_{2}\right)-\left(y_{1}+\mathrm{i} y_{2}\right)}\right) u(y) d y \\
= & -\frac{1}{\pi} \int_{\Omega \backslash B(x, \varepsilon)} \frac{\partial_{\bar{z}} u(y)}{\left(x_{1}+\mathrm{i} x_{2}\right)-\left(y_{1}+\mathrm{i} y_{2}\right)} d y \\
& +\frac{1}{2 \pi} \int_{\partial \Omega} \frac{u(y)}{\left(x_{1}+\mathrm{i} x_{2}\right)-\left(y_{1}+\mathrm{i} y_{2}\right)} \mathbf{n}(y) d s(y) \\
& +\frac{1}{2 \pi} \int_{\partial B(x, \varepsilon)} \frac{u(y)}{\left(x_{1}+\mathrm{i} x_{2}\right)-\left(y_{1}+\mathrm{i} y_{2}\right)} \frac{\left(x_{1}+\mathrm{i} x_{2}\right)-\left(y_{1}+\mathrm{i} y_{2}\right)}{|y-x|} d s(y) \\
:= & -A+B+C .
\end{aligned}
$$

However, we have

$$
C=\frac{1}{2 \pi} \int_{0}^{2 \pi} u(x+\varepsilon(\cos t, \sin t)) d t \longrightarrow u(x), \quad \text { when } \varepsilon \rightarrow 0 .
$$

By definition, if $\gamma:[0, \ell] \rightarrow \partial \Omega$ is a smooth arc-length parametrization of $\partial \Omega$ there holds

$$
\begin{aligned}
B & =-\frac{\mathrm{i}}{2 \pi} \int_{0}^{\ell} \frac{u(\gamma(t))}{\left(x_{1}+\mathrm{i} x_{2}\right)-\left(\gamma_{1}(t)+\mathrm{i} \gamma_{2}(t)\right)}\left(\gamma_{1}^{\prime}(t)+\mathrm{i} \gamma_{2}^{\prime}(t)\right) d t \\
& =-\frac{1}{2 \mathrm{i} \pi} \int_{\partial \Omega} \frac{u(\xi)}{\xi-\left(x_{1}+\mathrm{i} x_{2}\right)} d \xi=-\Phi_{\mathrm{h}}\left(\Gamma^{+} u\right)(x) .
\end{aligned}
$$

In particular, we obtain

$$
\begin{aligned}
\Phi_{\mathrm{h}}\left(\Gamma^{+} u\right)(x)= & \frac{1}{2 \pi} \int_{0}^{2 \pi} u(x+\varepsilon(\cos (t), \sin (t))) d t \\
& -\frac{1}{\pi} \int_{\Omega \backslash B(x, \varepsilon)} \frac{\partial_{\bar{z}} u(y)}{\left(x_{1}+\mathrm{i} x_{2}\right)-\left(y_{1}+\mathrm{i} y_{2}\right)} d y \\
= & \frac{1}{2 \pi} \int_{0}^{2 \pi} u(x+\varepsilon(\cos (t), \sin (t))) d t \\
& -\frac{1}{\pi} \int_{\mathbb{R}^{2}} \frac{\mathbb{1}_{\mathbb{R}^{2} \backslash B(0, \varepsilon)}(x-y)}{\left(x_{1}+\mathrm{i} x_{2}\right)-\left(y_{1}+\mathrm{i} y_{2}\right)}\left(\partial_{\bar{z}} u(y) \mathbb{1}_{\Omega}(y)\right) d y .
\end{aligned}
$$

Note that the linear form on $C_{0}^{\infty}\left(\mathbb{R}^{2}\right)$ defined by

$$
p . v .\left(\frac{1}{x_{1}+\mathrm{i} x_{2}}\right):=\varphi \in C_{0}^{\infty}\left(\mathbb{R}^{2}\right) \mapsto \lim _{\varepsilon \rightarrow 0} \int_{\mathbb{R}^{2}} \frac{\mathbb{1}_{\mathbb{R}^{2} \backslash B(0, \varepsilon)}(x)}{x_{1}+\mathrm{i} x_{2}} \varphi(x) d x \in \mathbb{C}
$$

\begin{tabular}{|l|l|l|l|l|l|}
\hline 220 & $\mathbf{3 9 5 9}$ & $\mathbf{B}$ & $\begin{array}{l}\text { Dispatch: 30/1/2021 } \\
\text { Total pages: 38 } \\
\text { Disk Received } \\
\text { Disk Used } \square\end{array}$ & $\begin{array}{l}\text { Journal: Commun. Math. Phys. } \\
\text { Not Used } \square \\
\text { Corrupted } \square \\
\text { Mismatch } \square\end{array}$ \\
\hline Jour. No & Ms. No.
\end{tabular}


belongs to $\mathcal{D}^{\prime}\left(\mathbb{R}^{2}\right)$. Remark that $\left(\partial_{\bar{z}} u \mathbb{1}_{\Omega}\right) \in \mathcal{D}^{\prime}\left(\mathbb{R}^{2}\right)$ and has compact support. Hence, p.v. $\left(\frac{1}{x_{1}+\mathrm{i} x_{2}}\right) *\left(\partial_{\bar{z}} u \mathbb{1}_{\Omega}\right) \in \mathcal{D}^{\prime}\left(\mathbb{R}^{2}\right)$ and taking the duality pairing with $\varphi \in C_{0}^{\infty}(\Omega)$ in (16) and $\varepsilon \rightarrow 0$ we get

$$
\left\langle\Phi_{\mathrm{h}}\left(\Gamma^{+} u\right)-u, \varphi\right\rangle_{\mathcal{D}^{\prime}(\Omega), \mathcal{D}(\Omega)}=\frac{1}{\pi}\left\langle p \cdot v \cdot\left(\frac{1}{x_{1}+\mathrm{i} x_{2}}\right) *\left(\partial_{\bar{z}} u \mathbb{1}_{\Omega}\right), \varphi\right\rangle_{\mathcal{D}^{\prime}\left(\mathbb{R}^{2}\right), \mathcal{D}\left(\mathbb{R}^{2}\right)} .
$$

Now, remark that $\mathcal{A}_{\mathrm{h}}^{2}(\Omega) \subset \operatorname{dom}\left(\partial_{\mathrm{h}}\right)$ and pick a sequence of $C^{\infty}(\bar{\Omega})$ functions $\left(v_{n}\right)_{n \in \mathbb{N}}$ which converges to $v \in \mathcal{A}_{\mathrm{h}}^{2}(\Omega)$ in the norm of $\operatorname{dom}\left(\partial_{\mathrm{h}}\right)$ when $n \rightarrow+\infty$. In particular, $\left(v_{n}\right)_{n \in \mathbb{N}}$ converges to $v$ and $\left(\partial_{\bar{z}} v_{n}\right) \mathbb{1}_{\Omega}$ converges to 0 when $n \rightarrow+\infty$ in $\mathcal{D}^{\prime}\left(\mathbb{R}^{2}\right)$. Using (17) for $u=v_{n}$ and letting $n \rightarrow+\infty$ we obtain that in $\mathcal{D}^{\prime}(\Omega)$ there holds $v=\Phi_{\mathrm{h}}\left(\Gamma^{+} v\right)$ where we have used the continuity of the map $\Phi_{\mathrm{h}} \circ \Gamma^{+}: \operatorname{dom}\left(\partial_{\mathrm{h}}\right) \rightarrow$ $L^{2}(\Omega)$, and the continuity of the convolution in $\mathcal{D}^{\prime}\left(\mathbb{R}^{2}\right)$. Now, remark that we also have $v=\Phi_{\mathrm{h}}\left(\Gamma^{+} v\right)$ in $\mathcal{A}_{\mathrm{h}}^{2}(\Omega)$ and taking the trace $\Gamma^{+}$on both side of this identity we get

$$
\Pi_{\mathrm{h}}^{+} \Gamma^{+} v=\Gamma^{+} v
$$

which implies $v=\Phi_{\mathrm{h}}\left(\Pi_{\mathrm{h}}^{+} \Gamma^{+} v\right)$ and proves the other inclusion.

We are now in a good position to prove Theorem 21.

Proof of Theorem 21. Proposition 25 is precisely the first statement of Theorem 21 thus, the only thing left to prove is the statement for the Hardy spaces. Now, recall that for $\sharp \in\{h, a h\}$, we have defined the Hardy spaces in (11) and that we want to prove

$$
\mathcal{H}_{\sharp}^{2}(\Omega)=\left\{\Phi_{\sharp}(f): f \in L^{2}(\partial \Omega), \Pi_{\sharp}^{-} f=0\right\} .
$$

Let $\mathcal{E}_{\sharp}$ be the set on the right-hand side, we prove both inclusions.

Inclusion $\mathcal{E}_{\sharp} \subset \mathcal{H}_{\sharp}^{2}(\Omega)$. Let $u=\Phi_{\sharp}(f) \in \mathcal{E}_{\sharp}$, by definition $u \in \mathcal{A}_{\sharp}^{2}$ et $\Gamma^{+} u=f \in$

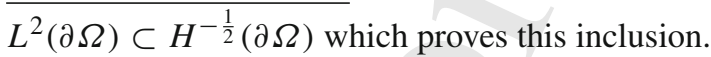

Inclusion $\mathcal{H}_{\sharp}^{2}(\Omega) \subset \mathcal{E}_{\sharp}$. Let $u \in \mathcal{H}_{\sharp}^{2}(\Omega)$. We know that in particular $u=\Phi_{\sharp}(f)$ for some $f \in H^{-\frac{1}{2}}(\partial \Omega)$ such that $\Pi_{\sharp}^{-} f=0$. But we have $\Gamma^{+} u=f \in L^{2}(\partial \Omega)$ which proves this inclusion and concludes the proof.

4.3. Explicit description of the domain of the maximal Wirtinger operators. In this paragraph, we prove the following description of the domains of the maximal Wirtinger operators introduced in Sect. 3. This description involves the Bergman spaces introduced in the beginning of Sect. 4.

Proposition 26. Let $\sharp \in\{\mathrm{h}$, ah\}. The following direct sum decomposition holds:

$$
\operatorname{dom}\left(\partial_{\sharp}\right)=\left\{u \in H^{1}(\Omega): \Pi_{\sharp}^{+} \Gamma^{+} u=0\right\} \dot{+} \mathcal{A}_{\sharp}^{2}(\Omega) .
$$

For $\sharp \in\{\mathrm{h}, \mathrm{ah}\}$, the range of the trace operator $\Gamma^{+}: \operatorname{dom}\left(\partial_{\sharp}\right) \rightarrow H^{-\frac{1}{2}}(\partial \Omega)$ is of crucial importance to prove Proposition 26. We describe its range now, thanks to the Szegő projectors introduced in (13) but first, we prove a regularization result.

\begin{tabular}{|l|l|l|l|l|l|}
\hline $\mathbf{2} 2 \mathbf{2 0}$ & $\mathbf{3 9 5 9}$ & $\mathbf{B}$ & $\begin{array}{l}\text { Dispatch: 30/1/2021 } \\
\text { Total pages: 38 } \\
\text { Disk Received } \\
\text { Disk Used } \square\end{array}$ & $\begin{array}{l}\text { Journal: Commun. Math. Phys. } \\
\text { Not Used } \square \\
\text { Corrupted } \square \\
\text { Mismatch } \square\end{array}$ \\
\hline Jour. No & Ms. No.
\end{tabular}


Lemma 27. Let $\sharp \in\left\{\mathrm{h}\right.$, ah\}. The operator $\Pi_{\sharp}^{-} \circ \Gamma^{+}$is a bounded linear operator from $\operatorname{dom}\left(\partial_{\sharp}\right)$ to $H^{\frac{1}{2}}(\partial \Omega)$.

Proof. Let $u \in \operatorname{dom}\left(\partial_{\mathrm{h}}\right)$ and $u_{n} \in C^{\infty}(\bar{\Omega})$ be a sequence converging to $u$ in the $\|\cdot\|_{\mathrm{h}^{-}}$ norm when $n \rightarrow+\infty$. Pick $f \in C^{\infty}(\partial \Omega)$, an integration by parts yields:

$$
\left\langle\Gamma^{+} u_{n}, \overline{\mathbf{n}} \Pi_{\mathrm{ah}}^{+} f\right\rangle_{L^{2}(\partial \Omega)}=2\left\langle\partial_{\bar{z}} u_{n}, \Phi_{\mathrm{ah}}(f)\right\rangle_{L^{2}(\Omega)} .
$$

It gives

$$
\left|\left\langle\Gamma^{+} u_{n}, \overline{\mathbf{n}} \Pi_{\mathrm{ah}}^{+} f\right\rangle_{L^{2}(\partial \Omega)}\right| \leq 2 c\left\|u_{n}\right\|_{\mathrm{h}}\|f\|_{H^{-\frac{1}{2}}(\partial \Omega)},
$$

for some $c>0$, where we have used Lemma 15 and Proposition 22. As in $L^{2}(\partial \Omega)$ there holds $S_{\mathrm{ah}}^{*}=-S_{\mathrm{h}}$ we get

$$
\left(\overline{\mathbf{n}} \Pi_{\mathrm{ah}}^{+}\right)^{*}=\Pi_{\mathrm{h}}^{-} \mathbf{n} .
$$

In particular, there holds

$$
\left|\left\langle\Gamma^{+} u_{n}, \overline{\mathbf{n}} \Pi_{\mathrm{ah}}^{+} f\right\rangle_{L^{2}(\partial \Omega)}\right|=\mid\left\langle\left(\Pi_{\mathrm{h}}^{-} \mathbf{n} \Gamma^{+} u_{n}, f\right\rangle_{L^{2}(\partial \Omega)}\right| \leq 2 c\left\|u_{n}\right\|_{\mathrm{h}}\|f\|_{H^{-\frac{1}{2}}(\partial \Omega)} .
$$

Letting $n \rightarrow+\infty$, we get $\Pi_{\mathrm{h}}^{-} \mathbf{n} \Gamma^{+} u \in H^{\frac{1}{2}}(\partial \Omega)$ and that $\Pi_{\mathrm{h}}^{-} \circ H_{\mathbf{n}} \circ \Gamma^{+}$is a linear bounded map from $H^{-\frac{1}{2}}(\partial \Omega)$ to $H^{\frac{1}{2}}(\partial \Omega)$. However, there holds

$$
\Pi_{\mathrm{h}}^{-} \Gamma^{+} u=\left(\overline{\mathbf{n}} \Pi_{\mathrm{h}}^{-} \mathbf{n}-\overline{\mathbf{n}}\left[\Pi_{\mathrm{h}}^{-}, \mathbf{n}\right]\right) \Gamma^{+} u=\overline{\mathbf{n}} \Pi_{\mathrm{h}}^{-} \mathbf{n} \Gamma^{+} u+\overline{\mathbf{n}}\left[S_{\mathrm{h}}, \mathbf{n}\right] \Gamma^{+} u .
$$

By (2) Proposition 12, $\left[S_{\mathrm{h}}, \mathbf{n}\right] \in \Psi_{\partial \Omega}^{-1}$ hence, it is a bounded operator from $H^{-\frac{1}{2}}(\partial \Omega)$ to $H^{\frac{1}{2}}(\partial \Omega)$. Finally, as the multiplication operator by $\overline{\mathbf{n}}$ is bounded in $H^{\frac{1}{2}}(\partial \Omega)$ we obtain the expected result.

The case $u \in \operatorname{dom}\left(\partial_{\mathrm{ah}}\right)$ is handled similarly.

We are now in a good position to describe the range of the trace operator $\Gamma^{+}$.

Corollary 28. Let $\sharp \in\{\mathrm{h}$, ah\}. There holds

$$
\operatorname{ran}\left(\Gamma^{+}\right)=\left\{f \in H^{-\frac{1}{2}}(\partial \Omega): \Pi_{\sharp}^{-} f \in H^{\frac{1}{2}}(\partial \Omega)\right\} .
$$

Proof. Let us start by proving the reverse inclusion. Let $f$ be in the set on right-hand side, there holds $f=\Pi_{\sharp}^{+} f+\Pi_{\sharp}^{-} f$. We know that there exists an extension operator $E^{+}$from $H^{\frac{1}{2}}(\partial \Omega)$ to $H^{1}(\Omega)$ such that $\Gamma^{+} E^{+} g=g$ for all $g \in H^{\frac{1}{2}}(\partial \Omega)$. Now, if $\Pi_{\sharp}^{-} f \in H^{\frac{1}{2}}(\partial \Omega)$, we set

$$
u:=\Phi_{\sharp}\left(\Pi_{\sharp}^{+} f\right)+E^{+}\left(\Pi_{\sharp}^{-} f\right) .
$$

It is easily seen that $u \in \operatorname{dom}\left(\partial_{\sharp}\right)$ and $\Gamma^{+} u=\Pi_{\sharp}^{+} f+\Pi_{\sharp}^{-} f=f$.

Now, let us prove the direct inclusion and pick $f \in \operatorname{ran}\left(\Gamma^{+}\right)$. We know that there exists $u \in \operatorname{dom}\left(\partial_{\sharp}\right)$ such that $f=\Gamma^{+} u$. In particular, by Lemma 27 we know that $\Pi_{\sharp}^{-} f=\Pi_{\sharp}^{-} \Gamma^{+} u \in H^{\frac{1}{2}}(\partial \Omega)$ which concludes the proof.

We are now able to prove Proposition 26.

\begin{tabular}{|l|l|l|l|l|l|}
\hline $\mathbf{2} 2 \mathbf{2 0}$ & $\mathbf{3 9 5 9}$ & $\mathbf{B}$ & $\begin{array}{l}\text { Dispatch: 30/1/2021 } \\
\text { Total pages: 38 } \\
\text { Disk Received } \\
\text { Disk Used } \square\end{array}$ & $\begin{array}{l}\text { Journal: Commun. Math. Phys. } \\
\text { Not Used } \square \\
\text { Corrupted } \square \\
\text { Mismatch } \square\end{array}$ \\
\hline
\end{tabular}


Proof of Proposition 26. First, let us prove that the sum is direct. Let $v=\Phi_{\sharp}(f)=u$ with $\Pi_{\sharp}^{-} f=0$ and $\Pi_{\sharp}^{+} \Gamma^{+} u=0$. Then, taking the traces we obtain:

$$
\Gamma^{+} v=\Pi_{\sharp}^{+} f=\Pi_{\sharp}^{-} \Gamma^{+} u,
$$

which implies $f=\Gamma^{+} u=0$. Consequently, $v=\Phi_{\sharp}(f)=0$.

Second, let us pick $v \in \operatorname{dom}\left(\partial_{\sharp}\right)$. There holds

$$
v=\Phi_{\sharp}\left(\Pi_{\sharp}^{+} \Gamma^{+} v\right)+v-\Phi_{\sharp}\left(\Pi_{\sharp}^{+} \Gamma^{+} v\right) .
$$

However, remark that $u:=v-\Phi_{\sharp}\left(\Pi_{\sharp}^{+} \Gamma^{+} v\right) \in \operatorname{dom}\left(\partial_{\sharp}\right)$ and satisfies $\Gamma^{+} u=\Pi_{\sharp}^{-} \Gamma^{+} v \in$ $H^{\frac{1}{2}}(\partial \Omega)$ by Lemma 27 . Hence, by Lemma 18 , we obtain $u \in H^{1}(\Omega)$ and $\Gamma^{+} u \in$ $\operatorname{ker} \Pi_{\sharp}^{+}=\operatorname{ran} \Pi_{\sharp}^{-}$, which concludes the proof.

\section{Variational Characterization of the Principal Eigenvalue}

The aim of this section is to prove Theorem 4. In Sect. 5.1 we describe precisely the $\operatorname{domains} \operatorname{dom}\left(q_{E}^{\Omega}\right)$ and $\operatorname{dom}\left(H_{E}^{\Omega}\right)$, where $H_{E}^{\Omega}$ is the unique self-adjoint operator associated with $q_{E}^{\Omega}$ via Kato's first representation theorem. In Sect. 5.2, we investigate the behavior of the map $E \in[0,+\infty) \mapsto \mu_{\Omega}(E)$. Finally, in Sect. 5.3, we prove Theorem 4 .

5.1. The quadratic form $q_{E}^{\Omega}$ and its associated self-adjoint operator $H_{E}^{\Omega}$. For $E>0$, recall that $q_{E}^{\Omega}$ is defined in (2) on the domain consisting of the closure of the $C^{\infty}(\bar{\Omega})$ functions with respect to the norm of the quadratic form

$$
N_{E}^{\Omega}(u):=\sqrt{\left\|\partial_{\bar{z}} u\right\|_{L^{2}(\Omega)}^{2}+\|u\|_{L^{2}(\Omega)}^{2}+E\|u\|_{L^{2}(\partial \Omega)}^{2}} .
$$

Remark that as defined, $q_{E}^{\Omega}$ is a closed, densely defined and bounded below quadratic form thus, by Kato's first representation theorem (see [25, Chap. VI, Thm. 2.1]), $q_{E}^{\Omega}$ is associated with a unique self-adjoint operator $H_{E}^{\Omega}$ acting in $L^{2}(\Omega)$ satisfying

$$
\operatorname{dom}\left(H_{E}^{\Omega}\right) \subset \operatorname{dom}\left(q_{E}^{\Omega}\right) .
$$

In this paragraph, we describe properties of the $\operatorname{domains} \operatorname{dom}\left(q_{E}^{\Omega}\right)$ and $\operatorname{dom}\left(H_{E}^{\Omega}\right)$ and start with the domain of the quadratic form $q_{E}^{\Omega}$.

Proposition 29. Let $E>0$. The form domain $\operatorname{dom}\left(q_{E}^{\Omega}\right)$ admits the following direct sum decomposition

$$
\operatorname{dom}\left(q_{E}^{\Omega}\right)=\left\{u \in H^{1}(\Omega): \Pi_{\mathrm{h}}^{+} \Gamma^{+} u=0\right\} \dot{+} \mathcal{H}_{\mathrm{h}}^{2}(\Omega) .
$$

Moreover, $\operatorname{dom}\left(q_{E}^{\Omega}\right)$ is continuously embedded in $H^{\frac{1}{2}}(\Omega)$.

\begin{tabular}{|l|l|l|l|l|l|}
\hline 220 & $\mathbf{3 9 5 9}$ & $\mathbf{B}$ & $\begin{array}{l}\text { Dispatch: 30/1/2021 } \\
\text { Total pages: 38 } \\
\text { Disk Received } \square \\
\text { Disk Used } \square\end{array}$ & $\begin{array}{l}\text { Journal: Commun. Math. Phys. } \\
\text { Not Used } \square \\
\text { Corrupted } \square \\
\text { Mismatch } \square\end{array}$ \\
\hline
\end{tabular}


Proof. Set $\mathcal{E}=\left\{u \in H^{1}(\Omega): \Pi_{\mathrm{h}}^{+} \Gamma^{+} u=0\right\}+\mathcal{H}_{\mathrm{h}}^{2}(\Omega)$ and remark that the sum is direct by the same arguments as in the proof of Proposition 26 . We prove the set equality by proving both inclusions.

Inclusion $\mathcal{E} \subset \operatorname{dom}\left(q_{E}^{\Omega}\right)$ Let $v:=u+\Phi_{\mathrm{h}}(f) \in \mathcal{E}$ and take $\left(u_{n}\right)_{n \in \mathbb{N}}$ and $\left(f_{n}\right)_{n \in \mathbb{N}}$ two sequences of functions such that

$$
\text { for all } n \in \mathbb{N} \quad u_{n} \in C^{\infty}(\bar{\Omega}), \quad f_{n} \in C^{\infty}(\partial \Omega)
$$

and

$$
\text { when } n \rightarrow+\infty \text { there holds }\left\|u_{n}-u\right\|_{H^{1}(\Omega)} \rightarrow 0, \quad\left\|f_{n}-f\right\|_{L^{2}(\partial \Omega)} \rightarrow 0 .
$$

By [10, Theorem 3.1.], we have $v_{n}:=u_{n}+\Phi_{\mathrm{h}}\left(f_{n}\right) \in C^{\infty}(\bar{\Omega})$ and for $E>0$, there exists $C>0$ such that there holds

$$
\begin{aligned}
& q_{E}^{\Omega}\left(v-v_{n}\right)+\left(E^{2}+1\right)\left\|v-v_{n}\right\|_{L^{2}(\Omega)}^{2} \\
& =4\left\|\partial_{\bar{z}}\left(u-u_{n}\right)\right\|_{L^{2}(\Omega)}^{2}+E\left\|\Gamma^{+}\left(u-u_{n}\right)+\Pi_{\mathrm{h}}^{+}\left(f-f_{n}\right)\right\|_{L^{2}(\partial \Omega)}^{2} \\
& \quad+\left\|\left(u-u_{n}\right)+\left(\Phi_{\mathrm{h}}\left(f-f_{n}\right)\right)\right\|_{L^{2}(\Omega)}^{2} \\
& \quad \leq C\left(\left\|u-u_{n}\right\|_{H^{1}(\Omega)}+\left\|f-f_{n}\right\|_{L^{2}(\partial \Omega)}\right),
\end{aligned}
$$

where we have used the mapping properties of $\Phi_{h}, \Gamma^{+}, \Pi_{\mathrm{h}}^{+}$and the continuity of the embedding of $L^{2}(\partial \Omega)$ into $H^{-\frac{1}{2}}(\partial \Omega)$. Letting $n \rightarrow+\infty$, we obtain that $v \in \operatorname{dom}\left(q_{E}^{\Omega}\right)$ and this inclusion is proved.

Inclusion $\operatorname{dom}\left(q_{E}^{\Omega}\right) \subset \mathcal{E}$ Let $\left(u_{n}\right)_{n \in \mathbb{N}} \in C^{\infty}(\bar{\Omega})^{\mathbb{N}}$ be a Cauchy sequence for the norm of the quadratic form $N_{E}^{\Omega}$. In particular, $\left(u_{n}\right)_{n \in \mathbb{N}}$ is a Cauchy sequence for the norm $\|\cdot\|_{\mathrm{h}}$ thus converges to $u \in \operatorname{dom}\left(\partial_{\mathrm{h}}\right)$ and by Lemma 15 , there holds $\| \Gamma^{+} u_{n}-$ $\Gamma^{+} u \|_{H^{-\frac{1}{2}}(\partial \Omega)} \rightarrow 0$ when $n \rightarrow+\infty$. Consequently, by Proposition 26 there exists $v \in\left\{w \in H^{1}(\Omega): \Pi_{\mathrm{h}}^{+} \Gamma^{+} w=0\right\}$ and $f \in H^{-\frac{1}{2}}(\partial \Omega)$ satisfying $\Pi_{\mathrm{h}}^{+} f=f$ such that $u=v+\Phi_{\mathrm{h}}(f)$. Let us prove that $f \in L^{2}(\partial \Omega)$ which will conclude the proof.

On the one hand as $\left(u_{n}\right)_{n \in \mathbb{N}} \in C^{\infty}(\bar{\Omega})^{\mathbb{N}}$ is a Cauchy sequence for the norm of the quadratic form $N_{E}^{\Omega},\left(\Gamma^{+} u_{n}\right)_{n \in \mathbb{N}}$ is a Cauchy sequence in $L^{2}(\partial \Omega)$ thus converges to some $g \in L^{2}(\partial \Omega)$. Necessarily $\Gamma^{+} u=g \in L^{2}(\partial \Omega)$. On the other hand in $H^{-\frac{1}{2}}(\partial \Omega)$ there holds

$$
f=\Gamma^{+} u-\Gamma^{+} v
$$

But as $\Gamma^{+} u \in L^{2}(\partial \Omega)$ and $\Gamma^{+} v \in H^{\frac{1}{2}}(\partial \Omega)$ we get $f \in L^{2}(\partial \Omega)$ and this inclusion is proved.

Let us consider the inclusion map

$$
\mathcal{I}:=\operatorname{dom}\left(q_{E}^{\Omega}\right) \rightarrow H^{\frac{1}{2}}(\Omega), \quad(\mathcal{I} u)=u .
$$

By Proposition 22 applied for $s=0$, this map is well defined. Consider $v_{n}:=u_{n}+$ $\Phi_{\mathrm{h}}\left(f_{n}\right) \in \operatorname{dom}\left(q_{E}^{\Omega}\right)$ which converges to $v$ in the norm of the quadratic form $q_{E}^{\Omega}$ and assume that $v_{n} \rightarrow w$ in the $H^{\frac{1}{2}}(\Omega)$-norm. In particular, as $v \in \operatorname{dom}\left(q_{E}^{\Omega}\right)$, there holds 
$v=u+\Phi_{\mathrm{h}}(f)$ for some $u \in H^{1}(\Omega)$ and $f \in L^{2}(\partial \Omega)$ as in the definition of $\mathcal{E}$. In particular, in $\mathcal{D}^{\prime}(\Omega)$ we obtain

$$
u+\Phi_{\mathrm{h}}(f)=w
$$

and as both terms belong to $H^{\frac{1}{2}}(\Omega)$, the closed graph theorem gives that $\mathcal{I}$ is continuous.

Because of the compact embedding of $H^{\frac{1}{2}}(\Omega)$ into $L^{2}(\Omega)$, an immediate corollary of Proposition 29 reads as follows.

Corollary 30. Let $E>0$, the operator $H_{E}^{\Omega}$ has compact resolvent and its spectrum consists of a non-decreasing sequence of eigengalues denoted $\left(\mu_{j}^{\Omega}(E)\right)_{j \geq 1}$. Moreover, there holds

$$
\mu_{j}^{\Omega}(E)=\inf _{\substack{F \subset \operatorname{dom}\left(q_{E}^{\Omega}\right) \\ \operatorname{dim} F=j}} \sup _{u \in F \backslash\{0\}} \frac{4 \int_{\Omega}\left|\partial_{\bar{z}} u\right|^{2} d x-E^{2} \int_{\Omega}|u|^{2} d x+E \int_{\partial \Omega}|u|^{2} d s}{\int_{\Omega}|u|^{2} d x} .
$$

Remark 31. For $E=0$, the counterpart of Propostion 29, would read

$$
\operatorname{dom}\left(q_{0}^{\Omega}\right)=\left\{u \in H^{1}(\Omega): \Pi_{\mathrm{h}}^{+} \Gamma^{+} u=0\right\} \dot{+} \mathcal{A}_{\mathrm{h}}^{2}(\Omega) .
$$

In particular, note that $\operatorname{dom}\left(q_{0}^{\Omega}\right)$ can not be included in any Sobolev space $H^{s}(\Omega)$, $(s>0)$. Indeed, for any Bergman function $u \in \mathcal{A}_{\mathrm{h}}^{2}(\Omega)$, there holds $q_{0}^{\Omega}(u)=0$ which implies that for all $j \geq 1$ we have $\mu_{j}^{\Omega}(0)=0$. Thus 0 is an eigenvalue of $H_{0}^{\Omega}$ of infinite multiplicity which would not be possible if we had $\operatorname{dom}\left(q_{0}^{\Omega}\right) \subset H^{s}(\Omega)$ because of the compact embedding of $H^{s}(\Omega)$ in $L^{2}(\Omega)$. This phenomena is reminiscent of what happens for the Dirac operator with zig-zag boundary conditions as discussed in [37].

We conclude this paragraph by a description of the domain of the operator $H_{E}^{\Omega}$.

Proposition 32. Let $E>0$, there holds:

$$
\operatorname{dom}\left(H_{E}^{\Omega}\right)=\left\{u \in H^{1}(\Omega): \partial_{\bar{z}} u \in H^{1}(\Omega) \text { and } \partial_{\bar{z}} u+\mathbf{n} \frac{E}{2} u=0 \text { on } \partial \Omega\right\} .
$$

Proof. Let $\mathcal{E}$ denote the set on the right-hand side of Proposition 32. The proof is performed proving both inclusions.

Inclusion $\operatorname{dom}\left(H_{E}^{\Omega}\right) \subset \mathcal{E}$

Let $u \in \operatorname{dom}\left(H_{E}^{\Omega}\right)$ and $v \in C_{0}^{\infty}(\Omega)$, there holds

$$
\begin{aligned}
\left\langle H_{E}^{\Omega} u, \bar{v}\right\rangle_{\mathcal{D}^{\prime}(\Omega), \mathcal{D}(\Omega)}=\left\langle H_{E}^{\Omega} u, v\right\rangle_{L^{2}(\Omega)}= & q_{E}^{\Omega}[u, v] \\
= & 4\left\langle\partial_{\bar{z}} u, \partial_{z} \bar{v}\right\rangle_{\mathcal{D}^{\prime}(\Omega), \mathcal{D}(\Omega)} \\
& -E^{2}\langle u, \bar{v}\rangle_{\mathcal{D}^{\prime}(\Omega), \mathcal{D}(\Omega)} \\
= & \left\langle\left(-\Delta-E^{2}\right) u, \bar{v}\right\rangle_{\mathcal{D}^{\prime}(\Omega), \mathcal{D}(\Omega)}
\end{aligned}
$$

\begin{tabular}{|l|l|l|l|l|l|}
\hline $\mathbf{2} 20$ & $\mathbf{3 9 5 9}$ & $\mathbf{B} \mathbf{3}$ & $\begin{array}{l}\text { Dispatch: 30/1/2021 } \\
\text { Total pages: 38 } \\
\text { Disk Received } \\
\text { Disk Used } \square\end{array}$ & $\begin{array}{l}\text { Journal: Commun. Math. Phys. } \\
\text { Not Used } \square \\
\text { Corrupted } \square \\
\text { Mismatch } \square\end{array}$ \\
\hline
\end{tabular}


where $q_{E}^{\Omega}[\cdot, \cdot]$ denotes the sesquilinear form associated with the quadratic form $q_{E}^{\Omega}$. Hence, in $L^{2}(\Omega)$, there holds $H_{E}^{\Omega} u=\left(-\Delta-E^{2}\right) u$. Remark that if $u \in \operatorname{dom}\left(H_{E}^{\Omega}\right)$ then $\partial_{\bar{z}} u \in \operatorname{dom}\left(\partial_{\mathrm{ah}}\right)$, in particular, by Green's Formula (10), for all $v \in C^{\infty}(\bar{\Omega})$ we get:

$$
\begin{aligned}
\left\langle H_{E}^{\Omega} u, v\right\rangle_{L^{2}(\Omega)}= & -4\left\langle\partial_{z}\left(\partial_{\bar{z}} u\right), v\right\rangle_{L^{2}(\Omega)}-E^{2}\langle u, v\rangle_{L^{2}(\Omega)} \\
= & 4\left\langle\partial_{\bar{z}} u, \partial_{\bar{z}} v\right\rangle_{L^{2}(\Omega)}-E^{2}\langle u, v\rangle_{L^{2}(\Omega)} \\
& -2\left\langle\overline{\mathbf{n}} \Gamma^{+} \partial_{\bar{z}} u, \Gamma^{+} v\right\rangle_{H^{-\frac{1}{2}}(\partial \Omega), H^{\frac{1}{2}}(\partial \Omega)} \\
= & q_{E}^{\Omega}[u, v]-\left\langle 2 \overline{\mathbf{n}} \Gamma^{+} \partial_{\bar{z}} u+E \Gamma^{+} u, \Gamma^{+} v\right\rangle_{H^{-\frac{1}{2}}(\partial \Omega), H^{\frac{1}{2}}(\partial \Omega)} .
\end{aligned}
$$

As $v \in \operatorname{dom}\left(q_{E}^{\Omega}\right)$ we necessarily have $\left\langle 2 \overline{\mathbf{n}} \Gamma^{+} \partial_{\bar{z}} u+E \Gamma^{+} u, \Gamma^{+} v\right\rangle_{H^{-\frac{1}{2}}(\partial \Omega), H^{\frac{1}{2}}(\partial \Omega)}=0$. As this is true for all $v \in C^{\infty}(\bar{\Omega})$ we obtain

$$
2 \overline{\mathbf{n}} \Gamma^{+} \partial_{\bar{z}} u+E \Gamma^{+} u=0, \quad \text { in } H^{-\frac{1}{2}}(\partial \Omega) .
$$

Taking the Szegő projectors in (18) we obtain

$$
\begin{aligned}
& \left(\Gamma^{+} \partial_{\bar{z}} u\right)+\frac{\mathbf{n}}{2} E \Gamma^{+} u=0 \\
& \Longleftrightarrow\left\{\begin{array}{l}
\Pi_{\mathrm{ah}}^{+}\left(\Gamma^{+}\left(\partial_{\bar{z}} u\right)\right)+\frac{E}{2} \Pi_{\mathrm{ah}}^{+} \mathbf{n} \Gamma^{+} u=0 \\
\Pi_{\mathrm{ah}}^{-}\left(\Gamma^{+}\left(\partial_{\bar{z}} u\right)\right)+\frac{E}{2} \Pi_{\mathrm{ah}}^{-} \mathbf{n} \Gamma^{+} u=0
\end{array}\right.
\end{aligned}
$$

Nevertheless, there holds

$$
\Pi_{\mathrm{ah}}^{-}=\Pi_{\mathrm{h}}^{+}-\frac{1}{2}\left(S_{\mathrm{h}}+S_{\mathrm{ah}}\right), \quad \Pi_{\mathrm{ah}}^{+}=\Pi_{\mathrm{h}}^{-}+\frac{1}{2}\left(S_{\mathrm{ah}}+S_{\mathrm{h}}\right) .
$$

In particular, we get

$$
\begin{aligned}
\Pi_{\mathrm{ah}}^{-}\left(\Gamma^{+}\left(\partial_{\bar{z}} u\right)\right) & =-\frac{E}{2} \Pi_{\mathrm{ah}}^{-}\left(\mathbf{n} \Gamma^{+} u\right) \\
& =-\frac{E}{2}\left(\mathbf{n} \Pi_{\mathrm{h}}^{+} \Gamma^{+} u+\left[\Pi_{\mathrm{h}}^{+}, \mathbf{n}\right] \Gamma^{+} u-\frac{1}{2}\left(S_{\mathrm{h}}+S_{\mathrm{ah}}\right)\left(\mathbf{n} \Gamma^{+} u\right)\right) \\
& =-\frac{E}{2}\left(\mathbf{n} \Pi_{\mathrm{h}}^{+} \Gamma^{+} u+\left[S_{\mathrm{h}}, \mathbf{n}\right] \Gamma^{+} u-\frac{1}{2}\left(S_{\mathrm{h}}+S_{\mathrm{ah}}\right)\left(\mathbf{n} \Gamma^{+} u\right)\right)
\end{aligned}
$$

It rewrites

$$
\Pi_{\mathrm{h}}^{+} \Gamma^{+} u=-\overline{\mathbf{n}}\left(\frac{2}{E} \Pi_{\mathrm{ah}}^{-} \Gamma^{+}\left(\partial_{\bar{z}} u\right)+\left[S_{\mathrm{h}}, \mathbf{n}\right] \Gamma^{+} u-\frac{1}{2}\left(S_{\mathrm{h}}+S_{\mathrm{ah}}\right)\left(\mathbf{n} \Gamma^{+} u\right)\right) .
$$

Remark that the right-hand side belongs to $H^{\frac{1}{2}}(\partial \Omega)$. This holds for the first term because of Lemma 27 and for the last two-terms because of Proposition 12. As $\Pi_{\mathrm{h}}^{-} \Gamma^{+} u \in$ $H^{\frac{1}{2}}(\partial \Omega)$ by Lemma 27 , we get $\Gamma^{+} u=\Pi_{\mathrm{h}}^{+} \Gamma^{+} u+\Pi_{\mathrm{h}}^{-} \Gamma^{+} u \in H^{\frac{1}{2}}(\partial \Omega)$ thus, by Lemma 18, $u \in H^{1}(\Omega)$. In particular $\Pi_{\mathrm{ah}}^{+}\left(\Gamma^{+}\left(\partial_{\bar{z}} u\right)\right)=-\frac{E}{2} \Pi_{\mathrm{ah}}^{+} \mathbf{n} \Gamma^{+} u \in H^{\frac{1}{2}}(\partial \Omega)$ and as $\Pi_{\mathrm{ah}}^{-} \Gamma^{+}\left(\partial_{\bar{z}} u\right) \in H^{\frac{1}{2}}(\partial \Omega)$ by Lemma 27 we obtain $\Gamma^{+} \partial_{\bar{z}} u=\Pi_{\mathrm{ah}}^{-} \Gamma^{+}\left(\partial_{\bar{z}} u\right)+$ 
$\Pi_{\mathrm{ah}}^{+} \Gamma^{+}\left(\partial_{\bar{z}} u\right) \in H^{\frac{1}{2}}(\partial \Omega)$ and by Lemma 18 we obtain $\partial_{\bar{z}} u \in H^{1}(\Omega)$. It concludes the proof of this inclusion.

Inclusion $\mathcal{E} \subset \operatorname{dom}\left(H_{E}^{\Omega}\right)$ Pick $u \in \mathcal{E}$. One easily sees that $\left(-\Delta-E^{2}\right) u \in L^{2}(\Omega)$, moreover for all $v \in \operatorname{dom}\left(q_{E}^{\Omega}\right)$, there holds

$$
q_{E}^{\Omega}[u, v]=\left\langle\left(-\Delta-E^{2}\right) u, v\right\rangle_{L^{2}(\Omega)}
$$

By definition of $H_{E}^{\Omega}$ it implies $u \in \operatorname{dom}\left(H_{E}^{\Omega}\right)$ and $H_{E}^{\Omega} u=\left(-\Delta-E^{2}\right) u$.

5.2. Concavity of the first min-max level. In this paragraph we investigate the behavior of the first min-max level $\mu^{\Omega}(E)$ with respect to the spectral parameter $E>0$. This behavior is illustrated in Fig. 3 for various bounded domains $\Omega$.

Proposition 33. The map $\mu^{\Omega}: E \geq 0 \mapsto \mu^{\Omega}(E)$ has the following properties.

1. $\mu^{\Omega}$ is a continuous and concave function on $\mathbb{R}_{+}$.

2. We have $\mu^{\Omega}(0)=0$ and there exists $E_{\star}^{\Omega}>0$ such that for all $E \in\left(0, E_{\star}^{\Omega}\right)$ there holds $\mu^{\Omega}(E)>0$.

3. Let $0<E_{1}<E_{2}$, there holds

$$
\mu^{\Omega}\left(E_{2}\right) \leq \frac{E_{2}}{E_{1}} \mu^{\Omega}\left(E_{1}\right)-E_{2}\left(E_{2}-E_{1}\right) .
$$

In particular, if $\mu^{\Omega}\left(E_{1}\right)=0$ (resp. $\mu^{\Omega}\left(E_{2}\right)=0$ ), there holds $\mu^{\Omega}\left(E_{2}\right)<0$ (resp. $\left.\mu^{\Omega}\left(E_{1}\right)>0\right)$.

Proof. As for all $u \in \operatorname{dom}\left(q_{E}^{\Omega}\right)$ the function $\left(E \geq 0 \mapsto q_{E}^{\Omega}(u)\right)$ is a continuous and concave, so is $\left(E \geq 0 \mapsto \mu^{\Omega}(E)\right)$ and Point (1) is proved.

Regarding Point (2), one observes that for all $u \in \operatorname{dom}\left(q_{E}^{\Omega}\right)$ there holds $q_{0}^{\Omega}(u) \geq 0$ and in particular $\mu^{\Omega}(0) \geq 0$. Now, for any $f \in L^{2}(\partial \Omega)$ we have $\Phi_{\mathrm{h}}(f) \in \operatorname{dom}\left(q_{E}^{\Omega}\right)$ and $q_{0}^{\Omega}(u)=0$ because $\Phi_{\mathrm{h}}(f)$ is holomorphic in $\Omega$. Consequently, there holds $\mu^{\Omega}(0)=0$.

To prove the second part of Point (2), let $u \in \operatorname{dom}\left(q_{E}^{\Omega}\right)$ and remark that

$$
q_{E}^{\Omega}(u)=(4-E)\left\|\partial_{\bar{z}} u\right\|_{L^{2}(\Omega)}^{2}-E^{2}\|u\|_{L^{2}(\Omega)}^{2}+E \mathfrak{Q}(u)
$$

where the quadratic form $\mathfrak{Q}$ is defined as

$$
\mathfrak{Q}(u)=\left\|\partial_{\bar{z}} u\right\|_{L^{2}(\Omega)}^{2}+\|u\|_{L^{2}(\partial \Omega)}^{2}, \quad \operatorname{dom}(\mathfrak{Q})=\operatorname{dom}\left(q_{E}^{\Omega}\right) .
$$

Now, remark that $\mathfrak{Q} \geq 0$ thus, by Kato's first representation theorem, there exists a unique self-adjoint operator $\mathfrak{H}$ such that $\operatorname{dom}(\mathfrak{H}) \subset \operatorname{dom}(\mathfrak{Q})$ and its spectrum is a sequence of non-decreasing eigenvalues because $\operatorname{dom}(\mathfrak{Q})=\operatorname{dom}\left(q_{E}^{\Omega}\right)$ is compactly embedded into $L^{2}(\Omega)$. Let $\lambda_{1}^{\Omega}$ be its smallest eigenvalue, we already know by the minmax principle that $\lambda_{1}^{\Omega} \geq 0$. Moreover, if $\lambda_{1}^{\Omega}=0$, for an associated eigenfunction $u$, we obtain $\mathfrak{Q}(u)=0$ which implies that $\partial_{\bar{z}} u=0$ hence $u$ is holomorphic with trace in $L^{2}(\partial \Omega)$. Consequently, $u$ belongs to $\mathcal{H}_{\mathrm{h}}^{2}(\Omega)$ and $u=\Phi_{\mathrm{h}}(f)$ for some $f \in L^{2}(\partial \Omega)$ such that $\Gamma^{+} u=f$. However, as $\mathfrak{Q}(u)=0$, we also obtain $\Gamma^{+} u=f=0$ which yields

\begin{tabular}{|l|l|l|l|l|l|}
\hline $\mathbf{2} 2 \mathbf{2 0}$ & $\mathbf{3 9 5 9}$ & $\mathbf{B}$ & $\begin{array}{l}\text { Dispatch: 30/1/2021 } \\
\text { Total pages: 38 } \\
\text { Disk Received } \\
\text { Disk Used } \square\end{array}$ & $\begin{array}{l}\text { Journal: Commun. Math. Phys. } \\
\text { Not Used } \square \\
\text { Corrupted } \\
\text { Mismatch } \square\end{array}$ \\
\hline Jour. No & Ms. No.
\end{tabular}


$u=0$ which is not possible because $u$ is an eigenfunction. It implies that $\lambda_{1}^{\Omega}>0$ and using the min-max principle in (19), we get for all $u \in \operatorname{dom}\left(q_{E}^{\Omega}\right)$ :

$$
q_{E}^{\Omega}(u) \geq(4-E)\left\|\partial_{\bar{z}} u\right\|_{L^{2}(\Omega)}^{2}-E^{2}\|u\|_{\Omega}^{2}+E \lambda_{1}^{\Omega}\|u\|_{L^{2}(\Omega)}^{2} .
$$

In particular, if $E<4$ we obtain

$$
q_{E}^{\Omega}(u) \geq E\left(\lambda_{1}^{\Omega}-E\right)\|u\|_{L^{2}(\Omega)}^{2}
$$

and the min-max principle yields

$$
\mu^{\Omega}(E) \geq E\left(\lambda_{1}^{\Omega}-E\right) .
$$

Thus, setting $E_{\star}^{\Omega}:=\min \left(4, \lambda_{1}^{\Omega}\right)$, for all $E \in\left(0, E_{\star}^{\Omega}\right)$, we have $\mu^{\Omega}(E)>0$.

Let us prove Point (3). Let $u \in \operatorname{dom}\left(q_{E}^{\Omega}\right)$ and $0<E_{1}<E_{2}$. There holds

$$
q_{E_{2}}^{\Omega}(u)=q_{E_{1}}^{\Omega}(u)-\left(E_{2}^{2}-E_{1}^{2}\right) \int_{\Omega}|u|^{2} d x+\left(E_{2}-E_{1}\right) \int_{\partial \Omega}|u|^{2} d s .
$$

Now, pick $u_{1}$ a normalized eigenfunction of $H_{E_{1}}^{\Omega}$ associated with the eigenvalue $\mu^{\Omega}\left(E_{1}\right)$. We have $q_{E_{1}}^{\Omega}\left(u_{1}\right)=\mu^{\Omega}\left(E_{1}\right)$ which implies

$$
\begin{aligned}
\int_{\partial \Omega}\left|u_{1}\right|^{2} d s & \leq \frac{1}{E_{1}}\left(4 \int_{\Omega}\left|\partial_{\bar{z}} u_{1}\right|^{2} d x+E_{1} \int_{\partial \Omega}\left|u_{1}\right|^{2} d s\right) \\
& =\frac{1}{E_{1}}\left(q_{E_{1}}^{\Omega}\left(u_{1}\right)+E_{1}^{2}\right) \\
& \leq \frac{E_{1}^{2}+\mu^{\Omega}\left(E_{1}\right)}{E_{1}} .
\end{aligned}
$$

Thus, evaluating (20) with $u=u_{1}$ we obtain

$$
q_{E_{2}}^{\Omega}\left(u_{1}\right) \leq \mu^{\Omega}\left(E_{1}\right)-\left(E_{2}^{2}-E_{1}^{2}\right)+\frac{E_{2}-E_{1}}{E_{1}}\left(E_{1}^{2}+\mu^{\Omega}\left(E_{1}\right)\right) .
$$

The min-max principle finally gives the sought inequality

$$
\begin{aligned}
\mu^{\Omega}\left(E_{2}\right) & \leq \mu^{\Omega}\left(E_{1}\right)-\left(E_{2}^{2}-E_{1}^{2}\right)+\frac{E_{2}-E_{1}}{E_{1}}\left(E_{1}^{2}+\mu^{\Omega}\left(E_{1}\right)\right) \\
& =\frac{E_{2}}{E_{1}} \mu^{\Omega}\left(E_{1}\right)-E_{2}\left(E_{2}-E_{1}\right) .
\end{aligned}
$$

Now, assume that $\mu^{\Omega}\left(E_{1}\right)=0$. It yields

$$
\mu^{\Omega}\left(E_{2}\right) \leq-E_{2}\left(E_{2}-E_{1}\right)<0 .
$$

Similarly, if $\mu^{\Omega}\left(E_{2}\right)=0$ we get

$$
0<E_{1}\left(E_{2}-E_{1}\right) \leq \mu^{\Omega}\left(E_{1}\right) .
$$

\begin{tabular}{|l|l|l|l|l|l|}
\hline 220 & $\mathbf{3 9 5 9}$ & $\mathbf{B}$ & $\begin{array}{l}\text { Dispatch: 30/1/2021 } \\
\text { Total pages: 38 } \\
\text { Disk Received } \\
\text { Disk Used } \square\end{array}$ & $\begin{array}{l}\text { Journal: Commun. Math. Phys. } \\
\text { Not Used } \square \\
\text { Corrupted } \square \\
\text { Mismatch } \square\end{array}$ \\
\hline
\end{tabular}


5.3. Proof of the variational principle. In our way to prove Theorem 4 we will need the following two propositions.

Proposition 34. Let $E>0$ be such that $\mu^{\Omega}(E)=0$ then $E \in \operatorname{Sp}_{\text {dis }}\left(D^{\Omega}\right)$.

Proof. Let $E>0$ be such that $\mu^{\Omega}(E)=0$ and consider a normalized associated eigenfunction $v \in \operatorname{dom}\left(H_{E}^{\Omega}\right)$. Set $u=\left(u_{1}, u_{2}\right)^{\top}=\left(v,-\frac{2 \mathrm{i}}{E} \partial_{\bar{z}} v\right)^{\top}$, by Proposition 32, $u \in H^{1}\left(\Omega, \mathbb{C}^{2}\right)$ and as $v \in \operatorname{dom}\left(H_{E}^{\Omega}\right)$, in $H^{\frac{1}{2}}(\partial \Omega)$ there holds

$$
\Gamma^{+}\left(\partial_{\bar{z}} v\right)+\mathbf{n} \frac{E}{2} \Gamma^{+} v=0 \Longleftrightarrow-2 E^{-1} \mathrm{i} \Gamma^{+}\left(\partial_{\bar{z}} v\right)=\mathrm{in} \Gamma^{+} u \Longleftrightarrow \Gamma^{+} u_{2}=\mathrm{in} \Gamma^{+} u_{1} .
$$

Hence, $\left(u_{1}, u_{2}\right)^{\top} \in \operatorname{dom}\left(D^{\Omega}\right)$ and there holds

$$
\begin{aligned}
D^{\Omega}\left(u_{1}, u_{2}\right)^{\top}=\left(\begin{array}{cc}
0 & -2 \mathrm{i} \partial_{z} \\
-2 \mathrm{i} \partial_{\bar{z}} & 0
\end{array}\right)\left(u_{1}, u_{2}\right)^{\top} & =\left(-2 \mathrm{i} \partial_{z} u_{2},-2 \mathrm{i} \partial_{\bar{z}} u_{1}\right)^{\top} \\
& =\left(-\frac{1}{E} \Delta u, E u_{2}\right)^{\top} \\
& =E\left(u_{1}, u_{2}\right)^{\top} .
\end{aligned}
$$

Hence, $E \in S p_{d i s}\left(D^{\Omega}\right)$ and it concludes the proof of Proposition 34 .

Proposition 35. Let $E \in S p_{d i s}\left(D^{\Omega}\right) \cap \mathbb{R}_{+}^{*}$ then $\mu^{\Omega}(E) \leq 0$.

Proof. Let $E \in \operatorname{Sp}_{\text {dis }}\left(D^{\Omega}\right) \cap \mathbb{R}_{+}^{*}$ and pick $u=\left(u_{1}, u_{2}\right)^{\top} \in \operatorname{dom}\left(D^{\Omega}\right)$ a normalized eigenfunction of $D^{\Omega}$ associated with $E$. We have

$$
\left\{\begin{array}{l}
D^{\Omega} u=E u \text { in } \Omega \\
u_{2}=\text { in } u_{1} \text { on } \partial \Omega .
\end{array}\right.
$$

In particular, we have $-2 \mathrm{i} \partial_{\bar{z}} u_{1}=E u_{2}$ and $\partial_{\bar{z}} u_{1} \in H^{1}(\Omega)$. It yields

$$
E u_{1}=-2 \mathrm{i} \partial_{z} u_{2}=-\frac{4}{E} \partial_{z} \partial_{\bar{z}} u_{1}
$$

Taking the scalar product with respect to $u_{1}$ on both side of the previous equation we get

$$
E^{2} \int_{\Omega}\left|u_{1}\right|^{2} d x=-4 \int_{\Omega}\left(\partial_{z} \partial_{\bar{z}} u_{1}\right) \overline{u_{1}} d x=4 \int_{\Omega}\left|\partial_{\bar{z}} u_{1}\right|^{2} d x-2 \int_{\partial \Omega} \overline{\mathbf{n}}\left(\partial_{\bar{z}} u_{1}\right) \overline{u_{1}} d s .
$$

Now, remark that on $\partial \Omega$, we have

$$
-\frac{2 \mathrm{i}}{E} \partial_{\bar{z}} u_{1}=u_{2}=\mathbf{i n} u_{1}
$$

which implies that on $\partial \Omega$

$$
2 \overline{\mathbf{n}} \partial_{\bar{z}} u_{1}+E u_{1}=0 \text {. }
$$

Hence, (21) becomes

$$
E^{2} \int_{\Omega}\left|u_{1}\right|^{2}=4 \int_{\Omega}\left|\partial_{\bar{z}} u_{1}\right|^{2} d x+E \int_{\partial \Omega}\left|u_{1}\right|^{2} d s
$$

which reads $q_{E}^{\Omega}\left(u_{1}\right)=0$ thus, the min-max principle gives $\mu^{\Omega}(E) \leq 0$. 
Now, we have all the tools to prove Theorem 4. The proof is performed proving each implication.

Proof of Theorem 4. By Proposition 35, we have $\mu^{\Omega}\left(E_{1}(\Omega)\right) \leq 0$. Assume that $\mu^{\Omega}\left(E_{1}(\Omega)\right)<$ 0 , by Proposition 33 we know that there exists $0<E<E_{1}(\Omega)$ such that $\mu^{\Omega}(E)=0$ which, by Proposition 34, implies $E \in S_{\text {dis }}\left(D^{\Omega}\right)$. It is not possible because, by definition of $E_{1}(\Omega), E \geq E_{1}(\Omega)$ consequently, we obtain $\mu^{\Omega}\left(E_{1}(\Omega)\right)=0$.

Let $E>0$ be such that $\mu^{\Omega}(E)=0$. By Proposition 34, $E \in \operatorname{Sp}_{\text {dis }}\left(D^{\Omega}\right)$ and necessarily $E \geq E_{1}(\Omega)$. If $E>E_{1}(\Omega)$, by Proposition 33, we obtain $\mu^{\Omega}\left(E_{1}(\Omega)\right)>0$ but by Proposition 35 we necessarily have $\mu^{\Omega}\left(E_{1}(\Omega)\right) \leq 0$ which implies that necessarily there holds $E=E_{1}(\Omega)$.

\section{Geometric Upper Bounds on the Spectral Gap}

The goal of this section is to prove Theorem 3 and this is discussed in Sect. 6.2. But first, in Sect. 6.1, we give a simple geometric upper bound on the spectral gap which illustrates how Theorem 4 can be used.

6.1. A simple upper bound. An immediate consequence of Theorem 4 reads as follows.

Proposition 36. Let $\Omega \subset \mathbb{R}^{2}$ be $C^{\infty}$ bounded and simply connected domain. There holds

$$
E_{1}(\Omega) \leq \frac{|\partial \Omega|}{|\Omega|}
$$

There is no reason for the above upper bound to be attained among Euclidean domains. However, the bound brings into play simple geometric quantities: the perimeter and the area of $\Omega$.

Proof. Let $E>0$ and $u \equiv 1$ the function constant to 1 in $\Omega$. As $u \in \operatorname{dom}\left(q_{E}^{\Omega}\right)$, by the min-max principle we obtain

$$
\mu^{\Omega}(E) \leq \frac{q_{E}^{\Omega}(u)}{\|u\|_{L^{2}(\Omega)}^{2}}=E\left(\frac{|\partial \Omega|}{|\Omega|}-E\right)
$$

So in $E_{\text {crit }}:=\frac{|\partial \Omega|}{|\Omega|}$ we get $\mu^{\Omega}\left(E_{\text {crit }}\right) \leq 0$ and by Proposition 33 we know that

$$
E_{1}(\Omega) \leq E_{\text {crit }}=\frac{|\partial \Omega|}{|\Omega|}
$$

\begin{tabular}{|l|l|l|l|l|l|}
\hline 220 & $\mathbf{3 9 5 9}$ & $\mathbf{B}$ & $\begin{array}{l}\text { Dispatch: 30/1/2021 } \\
\text { Total pages: 38 } \\
\text { Disk Received } \square \\
\text { Disk Used } \square\end{array}$ & $\begin{array}{l}\text { Journal: Commun. Math. Phys. } \\
\text { Not Used } \square \\
\text { Corrupted } \square \\
\text { Mismatch } \square\end{array}$ \\
\hline
\end{tabular}


6.2. A sharp upper bound. It turns out Theorem 3 is a consequence of the following result.

Theorem 37. Let $\Omega \subset \mathbb{R}^{2}$ be a $C^{\infty}$ bounded and simply connected domain. There holds

$$
E_{1}(\Omega) \leq \frac{|\partial \Omega|+\sqrt{|\partial \Omega|^{2}+8 \pi E_{1}(\mathbb{D})\left(E_{1}(\mathbb{D})-1\right)\left(\pi r_{i}^{2}+|\Omega|\right)}}{2\left(\pi r_{i}^{2}+|\Omega|\right)}
$$

with equality if and only if $\Omega$ is a disk.

Now, we have all the tools to prove Theorem 3.

Proof of Theorem 3. Using that $\pi r_{i}^{2} \leq|\Omega|$ and the isoperimetric inequality we obtain $4 \pi^{2} r_{i}^{2} \leq 4 \pi|\Omega| \leq|\partial \Omega|^{2}$. It gives

$$
|\partial \Omega|^{2}+8 \pi E_{1}(\mathbb{D})\left(E_{1}(\mathbb{D})-1\right)\left(\pi r_{i}^{2}+|\Omega|\right) \leq|\partial \Omega|^{2}\left(2 E_{1}(\mathbb{D})-1\right)^{2} .
$$

Note that in the above inequalities, we have equality if and only if $\Omega$ is a disk and combining this bound with the one of Theorem 37 we get Theorem 3 .

In the rest of this section we focus on proving Theorem 37 and assume, without loss of generality, the following.

(i) $0 \in \Omega$ is such that $r_{i}=\min _{x \in \partial \Omega}|x|$,

(ii) $f: \mathbb{D} \rightarrow \Omega$ is a conformal map such that $f(0)=0$ and we write

$$
f(z)=\sum_{n \geq 1} c_{n} z^{n}
$$

where $\left(c_{n}\right)_{n \geq 1}$ is a sequence of complex numbers.

Before going through the proof of Theorem 37, we gather in the following paragraph some known properties linking the geometry of $\Omega$ with the conformal map $f$.

6.2.1. Preliminaries The next proposition can be found in $[32, \S 3.10 .2]$ and relates the area of $\Omega$ with the conformal map $f$.

Proposition 38 (Area formula). There holds

$$
|\Omega|=\pi \sum_{n \geq 1} n\left|c_{n}\right|^{2} .
$$

The second proposition is a consequence of the Schwarz lemma (see Koebe's estimate in [21, Chap. I, Thm. 4.3]). It gives a relation between the first coefficient $c_{1}$ of the conformal map $f$ and the inradius $r_{i}$.

Proposition 39 (Koebe's estimate). There holds

$$
\left|f^{\prime}(0)\right|=\left|c_{1}\right| \geq r_{i} .
$$

Finally, the last geometric relation between the conformal map $f$ and the geometry of $\Omega$ we need to prove Theorem 37 is that the perimeter $|\partial \Omega|$ of $\Omega$ can be expressed as

$$
|\partial \Omega|=\int_{0}^{2 \pi}\left|f^{\prime}\left(e^{i \theta}\right)\right| d \theta
$$

Identity (22) is a simple consequence of the fact that $\left.f\right|_{\mathbb{S} 1}$ is a parametrization of $\partial \Omega$. 
6.2.2. Proof of the upper bound on the spectral gap To prove Theorem 37, we construct an adequate test function for $q_{E}^{\Omega}$ transplanting the eigenfunction of the unit disk $\mathbb{D}$ in the domain $\Omega$ using the conformal map $f$. We obtain an upper bound on $\mu^{\Omega}(E)$ which is a second order polynomial in the spectral parameter $E>0$ and with coefficients depending on the geometry of $\Omega$. It translates into an optimization problem for the spectral parameter $E>0$ that we solve in the last step of the proof.

Proof of Theorem 37. Let us go through all the steps of the proof.

Step 1 Let us denote by $J_{0}$ (resp. $J_{1}$ ) the Bessel function of the first kind of order 0 (resp. of order 1). For $x \in \mathbb{D}$, consider $u_{0}(x)=J_{0}\left(E_{1}(\mathbb{D})|x|\right) \in H^{1}(\mathbb{D}) \subset \operatorname{dom}\left(q_{E_{1}(\mathbb{D})}^{\Omega}\right)$. As explained in Remark $2 u(x)=\left(u_{0}(x), \mathrm{i} \frac{x_{1}+i x_{2}}{|x|} J_{1}\left(E_{1}(\mathbb{D})|x|\right)\right)^{\top}$ is an eigenfunction of $D^{\mathbb{D}}$ associated with $E_{1}(\mathbb{D})$. Theorem 4 implies

$$
\begin{aligned}
0=q_{E_{1}(\mathbb{D})}^{\mathbb{D}}\left(u_{0}\right)= & 2 \pi E_{1}(\mathbb{D})^{2} \int_{0}^{1} J_{1}\left(E_{1}(\mathbb{D}) r\right)^{2} r d r \\
& -2 \pi E_{1}(\mathbb{D})^{2} \int_{0}^{1} J_{0}\left(E_{1}(\mathbb{D}) r\right)^{2} r d r+2 \pi E_{1}(\mathbb{D}) J_{0}\left(E_{1}(\mathbb{D})\right)^{2} .
\end{aligned}
$$

Step 2 For $x=\left(x_{1}, x_{2}\right) \in \Omega$, consider $v_{0}\left(x_{1}, x_{2}\right)=u_{0}\left(f^{-1}\left(x_{1}+\mathrm{i} x_{2}\right)\right) \in H^{1}(\Omega) \subset$ $\operatorname{dom}\left(q_{E}^{\Omega}\right)$. By the min-max principle, there holds

$$
\mu^{\Omega}(E) \leq \frac{q_{E}^{\Omega}\left(v_{0}\right)}{\left\|v_{0}\right\|_{L^{2}(\Omega)}^{2}}=\frac{\left\|\nabla v_{0}\right\|_{L^{2}(\Omega)}^{2}+E\left\|v_{0}\right\|_{L^{2}(\partial \Omega)}^{2}}{\left\|v_{0}\right\|_{L^{2}(\Omega)}^{2}}-E^{2},
$$

where we have used that $v_{0}$ is real valued to ensure that $\left\|\nabla v_{0}\right\|_{L^{2}(\Omega)}=4\left\|\partial_{\bar{z}} v_{0}\right\|_{L^{2}(\Omega)}$. Step 3 Now, as $f$ is a conformal map, we know that

$$
\left\|\nabla v_{0}\right\|_{L^{2}(\Omega)}^{2}=\left\|\nabla u_{0}\right\|_{L^{2}(\mathbb{D})}^{2}=2 \pi E_{1}(\mathbb{D})^{2} \int_{0}^{1} J_{1}\left(E_{1}(\mathbb{D}) r\right)^{2} r d r .
$$

Using (22), we obtain

$$
\left\|v_{0}\right\|_{L^{2}(\partial \Omega)}^{2}=\int_{0}^{2 \pi}\left|v_{0}\left(f\left(e^{\mathrm{i} \theta}\right)\right)\right|^{2}\left|f^{\prime}\left(e^{\mathrm{i} \theta}\right)\right| d \theta=J_{0}\left(E_{1}(\mathbb{D})\right)^{2}|\partial \Omega| .
$$

Finally, the last integral reads

$$
\begin{aligned}
\left\|v_{0}\right\|_{L^{2}(\Omega)}^{2} & =\int_{0}^{1} \int_{0}^{2 \pi}\left|u_{0}(r)\right|^{2}\left|f^{\prime}\left(r e^{\mathrm{i} \theta}\right)\right|^{2} r d r d \theta \\
& =\int_{0}^{1}\left|u_{0}(r)\right|^{2}\left(\int_{0}^{2 \pi}\left|\sum_{n \geq 1} n c_{n} r^{n-1} e^{\mathrm{i}(n-1) \theta}\right|^{2} d \theta\right) r d r \\
& =2 \pi \sum_{n \geq 1} n\left|c_{n}\right|^{2} M_{n},
\end{aligned}
$$

where we have used Parseval identity. Here, for $n \geq 1$ we have set $M_{n}:=n \int_{0}^{1} J_{0}$ $\left(E_{1}(\mathbb{D}) r\right)^{2} r^{2 n-1} d r$.

\begin{tabular}{|l|l|l|l|l|l|}
\hline $\mathbf{2} 20$ & $\mathbf{3 9 5 9}$ & $\mathbf{B}$ & $\begin{array}{l}\text { Dispatch: 30/1/2021 } \\
\text { Total pages: 38 } \\
\text { Disk Received } \\
\text { Disk Used } \square\end{array}$ & $\begin{array}{l}\text { Journal: Commun. Math. Phys. } \\
\text { Not Used } \square \\
\text { Corrupted } \square \\
\text { Mismatch } \square\end{array}$ \\
\hline Jour. No & Ms. No.
\end{tabular}


Step 4 Taking into account (25), (26) and (27), (24) becomes

$$
\begin{aligned}
\mu^{\Omega}(E) \leq & 2 \pi E_{1}(\mathbb{D})^{2} \frac{\int_{0}^{1} J_{1}\left(E_{1}(\mathbb{D}) r\right)^{2} r d r}{2 \pi \sum_{n \geq 1} n\left|c_{n}\right|^{2} M_{n}}-E^{2} \\
& +E \frac{J_{0}\left(E_{1}(\mathbb{D})\right)^{2}|\partial \Omega|}{2 \pi \sum_{n \geq 1} n\left|c_{n}\right|^{2} M_{n}} .
\end{aligned}
$$

Let us find a lower bound on the sequence $\left(M_{n}\right)_{n \geq 1}$. Using first an integration by parts we find

$$
M_{n}=\frac{1}{2} J_{0}\left(E_{1}(\mathbb{D})\right)^{2}+\frac{E_{1}(\mathbb{D})}{2} \int_{0}^{1} J_{0}\left(E_{1}(\mathbb{D}) r\right) J_{1}\left(E_{1}(\mathbb{D}) r\right) r^{2 n} d r .
$$

In particular, for $n=1$ it gives

$$
\begin{aligned}
M_{1} & =\int_{0}^{1} J_{0}\left(E_{1}(\mathbb{D}) r\right)^{2} r d r=J_{0}\left(E_{1}(\mathbb{D})\right)^{2} \\
& =E_{1}(\mathbb{D}) \int_{0}^{1} J_{0}\left(E_{1}(\mathbb{D}) r\right) J_{1}\left(E_{1}(\mathbb{D}) r\right) r^{2} d r .
\end{aligned}
$$

Now, for $n \geq 1$, one notices that $h_{1}:=\left(r \mapsto\left(J_{0} J_{1}\right)\left(E_{1}(\mathbb{D}) r\right) r^{2}\right)$ and $h_{2}:=(r \mapsto$ $\left.r^{2 n-2}\right)$ are non-decreasing functions on $[0,1]$ and by Chebyschev's inequality for nondecreasing functions, we obtain

$$
M_{n} \geq \frac{1}{2} M_{1}+\frac{1}{2} M_{1} \int_{0}^{1} r^{2 n-2} d r=\frac{n}{2 n-1} M_{1} .
$$

In particular, we have

$$
\begin{aligned}
2 \pi \sum_{n \geq 1} n\left|c_{n}\right|^{2} M_{n} & \geq J_{0}\left(E_{1}(\mathbb{D})\right)^{2}\left(2 \pi\left|c_{1}\right|^{2}+2 \pi \sum_{n \geq 2} \frac{n^{2}}{2 n-1}\left|c_{n}\right|^{2}\right) \\
& \geq J_{0}\left(E_{1}(\mathbb{D})\right)^{2}\left(2 \pi\left|c_{1}\right|^{2}+\pi \sum_{n \geq 2} n\left|c_{n}\right|^{2}\right) \\
& =J_{0}\left(E_{1}(\mathbb{D})\right)^{2}\left(\pi\left|c_{1}\right|^{2}+|\Omega|\right) \\
& \geq J_{0}\left(E_{1}(\mathbb{D})\right)^{2}\left(\pi\left|r_{i}\right|^{2}+|\Omega|\right),
\end{aligned}
$$

where we have used Proposition 38 and Proposition 39. Remark that in the first two inequalities above we have equality if and only if $c_{n}=0$ for all $n \geq 2$. Similarly, in the last equality, we have equality if and only if $\left|c_{1}\right|=r_{i}$. In particuliar there is equality in the above inequalities if and only if $f(z)=c_{1} z$ and $\Omega$ is a disk centered in 0 of radius $r_{i}$.

\begin{tabular}{|l|l|l|l|l|l|}
\hline $\mathbf{2} 2 \mathbf{2 0}$ & $\mathbf{3 9 5 9}$ & $\mathbf{B}$ & $\begin{array}{l}\text { Dispatch: 30/1/2021 } \\
\text { Total pages: 38 } \\
\text { Disk Received } \\
\text { Disk Used } \square\end{array}$ & $\begin{array}{l}\text { Journal: Commun. Math. Phys. } \\
\text { Not Used } \square \\
\text { Corrupted } \square \\
\text { Mismatch } \square\end{array}$ \\
\hline Jour. No & Ms. No.
\end{tabular}


Combining (23) and (30) in (28), we obtain

$\mu^{\Omega}(E) \leq-E^{2}$

$$
+\frac{2 \pi E_{1}(\mathbb{D})^{2} \int_{0}^{1} J_{0}\left(E_{1}(\mathbb{D}) r\right)^{2} r d r+J_{0}\left(E_{1}(\mathbb{D})\right)^{2}\left(E|\partial \Omega|-2 \pi E_{1}(\mathbb{D})\right)}{J_{0}\left(E_{1}(\mathbb{D})\right)^{2}\left(\pi r_{i}^{2}+|\Omega|\right)} .
$$

Using (29), we obtain

$$
\begin{aligned}
\mu^{\Omega}(E) & \leq-E^{2}+\frac{2 \pi E_{1}(\mathbb{D})^{2}+\left(E|\partial \Omega|-2 \pi E_{1}(\mathbb{D})\right)}{\pi r_{i}^{2}+|\Omega|} \\
& =\frac{\left(2 \pi E_{1}(\mathbb{D})^{2}-\left(\pi r_{i}^{2}+|\Omega|\right) E^{2}\right)+\left(E|\partial \Omega|-2 \pi E_{1}(\mathbb{D})\right)}{\pi r_{i}^{2}+|\Omega|} \\
& =\frac{P(E)}{\pi r_{i}^{2}+|\Omega|},
\end{aligned}
$$

where we have set $P(E):=-E^{2}\left(\pi r_{i}^{2}+|\Omega|\right)+E|\partial \Omega|+2 \pi E_{1}(\mathbb{D})\left(E_{1}(\mathbb{D})-1\right)$.

Step 5 Remark that by (1), there holds $E_{1}(\mathbb{D})-1 \geq \sqrt{2}-1>0$. In particular, the discriminant of $P$ satisfies

$$
\delta(P):=|\partial \Omega|^{2}+8 \pi E_{1}(\mathbb{D})\left(E_{1}(\mathbb{D})-1\right)\left(\pi r_{i}^{2}+|\Omega|\right)>0 .
$$

Thus, $P$ has two real roots and as $P(0)>0$, the only positive root is

$$
E_{\text {crit }}:=\frac{|\partial \Omega|+\sqrt{|\partial \Omega|^{2}+8 \pi E_{1}(\mathbb{D})\left(E_{1}(\mathbb{D})-1\right)\left(\pi r_{i}^{2}+|\Omega|\right)}}{2\left(\pi r_{i}^{2}+|\Omega|\right)} .
$$

One obtains $\mu^{\Omega}\left(E_{\text {crit }}\right) \leq \frac{P\left(E_{\text {crit }}\right)}{\pi r_{i}^{2}+|\Omega|}=0$ and by Proposition 33 and Theorem 4 we get

$$
E_{1}(\mathbb{D}) \leq E_{\text {crit }}
$$

which is precisely Theorem 37 .

\section{About the Faber-Krahn Conjecture}

In this section we discuss how the variational formulation established in Theorem 4 can be used to investigate Conjecture 1. We start by discussing a new Faber-Krahn conjecture for the operator $H_{E}^{\Omega}$ introduced in Sect. 5.1 and show that the problem implies the Bossel-Daners inequality for the Robin Laplacian (see $[13,15]$ ).

This new conjecture reads as follows.

Conjecture 40. Let $\Omega \subset \mathbb{R}^{2}$ be $C^{\infty}$ bounded and simply connected domain. For all $E>0$, there holds

$$
\mu^{\Omega}(E) \geq \frac{\pi}{|\Omega|} \mu \mathbb{D}\left(\sqrt{\frac{|\Omega|}{\pi}} E\right) .
$$

Moreover, there is equality in the above inequality if and only if $\Omega$ is a disk.

\begin{tabular}{|l|l|l|l|l|l|}
\hline $\mathbf{2} 2 \mathbf{2 0}$ & $\mathbf{3 9 5 9}$ & $\mathbf{B}$ & $\begin{array}{l}\text { Dispatch: 30/1/2021 } \\
\text { Total pages: 38 } \\
\text { Disk Received } \\
\text { Disk Used } \square\end{array}$ & $\begin{array}{l}\text { Journal: Commun. Math. Phys. } \\
\text { Not Used } \square \\
\text { Corrupted } \square \\
\text { Mismatch } \square\end{array}$ \\
\hline Jour. No & Ms. No.
\end{tabular}


Conjecture 40 implies Conjecture 1 as can be seen evaluating (31) in $E=E_{1}(\Omega)$ and using Proposition 33 as well as Theorem 4.

It is remarkable that (31) scales as the Bossel-Daners inequality :

$$
\lambda_{\mathrm{Rob}}^{\Omega}(E) \geq \frac{\pi}{|\Omega|} \lambda_{\mathrm{Rob}}^{\mathbb{D}}\left(\sqrt{\frac{|\Omega|}{\pi}} E\right),
$$

with equality if and only if $\Omega$ is a disk. Here $\lambda_{\operatorname{Rob}}^{\Omega}(E)$ is given for $E>0$ by

$$
\lambda_{\operatorname{Rob}}^{\Omega}(E):=\inf _{u \in C^{\infty}(\bar{\Omega}) \backslash\{0\}} \frac{\|\nabla u\|_{L^{2}(\Omega)}^{2}+E \int_{\partial \Omega}|u|^{2} d s}{\|u\|_{L^{2}(\Omega)}^{2}} .
$$

Actually, one can prove the following proposition.

Proposition 41. Conjecture 1 implies the Bossel-Daners inequality (32).

Proof. Assume that Conjecture 40 holds. By separation of variables, let us start by remarking that for all $E>0$, if $u \in \operatorname{dom}\left(H_{E}^{\mathbb{D}}\right)$ is a normalized eigenfunction associated with $\mu^{\mathbb{D}}(E)$ then $u$ can be picked real valued. Hence, we get

$$
\begin{aligned}
\mu^{\mathbb{D}}(E) & =\inf _{v \in C^{\infty}(\overline{\mathbb{D}}, \mathbb{R})} \frac{\|\nabla v\|_{L^{2}(\mathbb{D})}^{2}-E^{2}\|v\|_{L^{2}(\mathbb{D})}^{2}+E \int_{\partial \mathbb{D}}|v|^{2} d s}{\|v\|_{L^{2}(\mathbb{D})}^{2}} \\
& =\lambda \operatorname{Rob}_{\operatorname{Rob}}(E)-E^{2} .
\end{aligned}
$$

Now, we remark that for any domain $\Omega$ there holds

$$
\begin{aligned}
\lambda_{\mathrm{Rob}}^{\Omega}(E)-E^{2} & =\inf _{v \in C^{\infty}(\bar{\Omega}, \mathbb{R}) \backslash\{0\}} \frac{\|\nabla v\|_{L^{2}(\Omega)}^{2}-E^{2}\|v\|_{L^{2}(\Omega)}^{2}+E \int_{\partial \Omega}|v|^{2} d s}{\|v\|_{L^{2}(\Omega)}^{2}} \\
& =\inf _{v \in C^{\infty}(\bar{\Omega}, \mathbb{R}) \backslash\{0\}} \frac{4\left\|\partial_{\bar{z}} v\right\|_{L^{2}(\Omega)}^{2}-E^{2}\|v\|_{L^{2}(\Omega)}^{2}+E \int_{\partial \Omega}|v|^{2} d s}{\|v\|_{L^{2}(\Omega)}^{2}} \\
& \geq \inf _{\left.v \in \operatorname{dom}\left(q_{E}^{\Omega}\right)\right) \backslash\{0\}} \frac{4\left\|\partial_{\bar{z}} v\right\|_{L^{2}(\Omega)}^{2}-E^{2}\|v\|_{L^{2}(\Omega)}^{2}+E \int_{\partial \Omega}|v|^{2} d s}{\|v\|_{L^{2}(\Omega)}^{2}} \\
& =\mu^{\Omega}(E) .
\end{aligned}
$$

Hence, using (33) and (34), we get

$$
\lambda_{\mathrm{Rob}}^{\Omega}(E)-E^{2} \geq \mu^{\Omega}(E) \geq \frac{\pi}{|\Omega|} \mu \mathbb{D}\left(\sqrt{\frac{|\Omega|}{\pi}} E\right)=\frac{\pi}{|\Omega|} \lambda_{\operatorname{Rob}}^{\mathbb{D}}\left(\sqrt{\frac{|\Omega|}{\pi}} E\right)-E^{2} .
$$

If $\Omega$ is a disk, all the above inequalities are equalities. Else, we obtain

$$
\lambda_{\mathrm{Rob}}^{\Omega}(E)>\frac{\pi}{|\Omega|} \lambda \underset{\operatorname{Rob}}{\mathbb{D}}\left(\sqrt{\frac{|\Omega|}{\pi}} E\right),
$$

which is precisely the Bossel-Daners inequality (32).

\begin{tabular}{|l|l|l|l|l|l|}
\hline 220 & $\mathbf{3 9 5 9}$ & $\mathbf{B}$ & $\begin{array}{l}\text { Dispatch: 30/1/2021 } \\
\text { Total pages: 38 } \\
\text { Disk Received } \\
\text { Disk Used } \square\end{array}$ & $\begin{array}{l}\text { Journal: Commun. Math. Phys. } \\
\text { Not Used } \square \\
\text { Corrupted } \\
\text { Mismatch } \square\end{array}$ \\
\hline Jour. No & Ms. No.
\end{tabular}




\section{Numerics}

The goal of this section is to illustrate numerically some theoretical results discussed in the previous sections and to support the validity of Conjecture 1.

In Sect. 8.1, we discuss the two numerical schemes we have employed in Sect. 8.2 in order to study the principal eigenvalue of the Dirac operator with infinite mass boundary conditions in various domains $\Omega$. We also discuss the structure of the associated eigenfunctions.

\subsection{Numerical methods. In this paragraph we present a brief description of the numer-} ical methods that we use in this work.

We have implemented two different numerical approaches, respectively to calculate the eigenvalues of the Dirac operator with infinite mass boundary conditions, directly from the formulation of the eigenvalue problem and to solve the minimization problem associated with the non-linear variational characterization (3), defining $\mu^{\Omega}(E)$.

The eigenvalues of the Dirac operator with infinite mass boundary conditions are calculated using a numerical method based on Radial Basis Functions (RBF) (see eg. [20, 24]). We have chosen a set of $R B F$ centers $y_{1}, \ldots, y_{N} \in \mathbb{R}^{2}$, for some $N \in \mathbb{N}$, which are generated by a node repel algorithm (see [2] for details). The eigenfunction $u=\left(u_{1}, u_{2}\right)^{\top}$ is defined in $H^{1}\left(\Omega, \mathbb{C}^{2}\right)$ and we use the notation $u_{1}=v_{1}+\mathrm{i} w_{1}$ and $u_{2}=v_{2}+\mathrm{i} w_{2}$, where $v_{1}, w_{1}$ and $v_{2}, w_{2}$ are the real and imaginary parts of $u_{1}$ and $u_{2}$, respectively. The RBF numerical approximation for each of these functions is defined by

$$
\begin{array}{ll}
v_{1}(x)=\sum_{j=1}^{N} \alpha_{j}^{(1)} \phi_{j}(x), & w_{1}(x)=\sum_{j=1}^{N} \beta_{j}^{(1)} \phi_{j}(x), \\
v_{2}(x)=\sum_{j=1}^{N} \alpha_{j}^{(2)} \phi_{j}(x), & w_{2}(x)=\sum_{j=1}^{N} \beta_{j}^{(2)} \phi_{j}(x),
\end{array}
$$

where $\phi_{j}(x)=\phi\left(\left|x-y_{j}\right|\right)$, for some function $\phi: \mathbb{R}_{0}^{+} \rightarrow \mathbb{R}$. Several $R B F$ functions can be considered (eg. [2,20]), but in this work we consider the multiquadric one $\phi(r)=$ $\sqrt{1+(\epsilon r)^{2}}$, for some $\epsilon>0$.

The eigenvalue problem for the Dirac operator with infinite mass boundary conditions can be written as

$$
\left\{\begin{array}{cl}
-\frac{\partial v_{2}}{\partial x_{2}}+\frac{\partial w_{2}}{\partial x_{1}}+\mathrm{i}\left(-\frac{\partial v_{2}}{\partial x_{1}}-\frac{\partial w_{2}}{\partial x_{2}}\right)=E\left(v_{1}+\mathrm{i} w_{1}\right) & \text { in } \Omega \\
\frac{\partial w_{1}}{\partial x_{1}}+\frac{\partial v_{1}}{\partial x_{2}}+\mathrm{i}\left(-\frac{\partial v_{1}}{\partial x_{1}}+\frac{\partial w_{1}}{\partial x_{2}}\right)=E\left(v_{2}+\mathrm{i} w_{2}\right) & \text { in } \Omega \\
\left(v_{2}+\mathrm{i} w_{2}\right)=\mathrm{i}\left(n_{1}+\mathrm{i} n_{2}\right)\left(v_{1}+\mathrm{i} w_{1}\right) & \text { on } \partial \Omega
\end{array}\right.
$$

and splitting in real and imaginary parts we have

$$
\left\{\begin{array}{cl}
-\frac{\partial v_{2}}{\partial x_{2}}+\frac{\partial w_{2}}{\partial x_{1}}=E v_{1} & \text { in } \Omega \\
-\frac{\partial v_{2}}{\partial x_{1}}-\frac{\partial w_{2}}{\partial x_{2}}=E w_{1} & \text { in } \Omega \\
\frac{\partial w_{1}}{\partial x_{1}}+\frac{\partial v_{1}}{\partial x_{2}}=E v_{2} & \text { in } \Omega \\
-\frac{\partial v_{1}}{\partial x_{1}}+\frac{\partial w_{1}}{\partial x_{2}}=E w_{2} & \text { in } \Omega \\
v_{2}=-n_{1} w_{1}-n_{2} v_{1} & \text { on } \partial \Omega \\
w_{2}=n_{1} v_{1}-n_{2} w_{1} & \text { on } \partial \Omega
\end{array}\right.
$$

\begin{tabular}{|l|l|l|l|l|l|}
\hline $\mathbf{2} 2 \mathbf{2 0}$ & $\mathbf{3 9 5 9}$ & $\mathbf{B}$ & $\begin{array}{l}\text { Dispatch: 30/1/2021 } \\
\text { Total pages: 38 } \\
\text { Disk Received } \\
\text { Disk Used } \square\end{array}$ & $\begin{array}{l}\text { Journal: Commun. Math. Phys. } \\
\text { Not Used } \square \\
\text { Corrupted } \square \\
\text { Mismatch } \square\end{array}$ \\
\hline Jour. No & Ms. No.
\end{tabular}


These equations are imposed at a discrete set of interior and boundary points. We consider $M^{\partial \Omega} \in \mathbb{N}$ points $p_{1}, \ldots, p_{M^{\partial} \Omega}$ uniformly distributed on $\partial \Omega$ and $M^{\Omega} \in \mathbb{N}$ points $q_{1}, \ldots, q_{M^{\Omega}}$ located at a grid defined on $\Omega$. Then, we calculate the matrices

$$
\begin{gathered}
\mathbf{M}^{\Omega}=\left[\begin{array}{ccc}
\phi_{1}\left(q_{1}\right) & \cdots & \phi_{N}\left(q_{1}\right) \\
\vdots & \ddots & \vdots \\
\phi_{1}\left(q_{M^{\Omega}}\right) & \cdots & \phi_{N}\left(q_{M^{\Omega}}\right)
\end{array}\right], \quad \mathbf{M}_{1}^{\Omega}=\left[\begin{array}{ccc}
\partial_{1} \phi_{1}\left(q_{1}\right) & \cdots & \partial_{1} \phi_{N}\left(q_{1}\right) \\
\vdots & \ddots & \vdots \\
\partial_{1} \phi_{1}\left(q_{M^{\Omega}}\right) & \cdots & \partial_{1} \phi_{N}\left(q_{\left.M^{\Omega}\right)}\right.
\end{array}\right], \\
\mathbf{M}_{2}^{\Omega}=\left[\begin{array}{ccc}
\partial_{2} \phi_{1}\left(q_{1}\right) & \cdots & \partial_{2} \phi_{N}\left(q_{1}\right) \\
\vdots & \ddots & \vdots \\
\partial_{2} \phi_{1}\left(q_{M^{\Omega}}\right) & \cdots & \partial_{2} \phi_{N}\left(q_{\left.M^{\Omega}\right)}\right.
\end{array}\right], \mathbf{M}^{\partial \Omega}=\left[\begin{array}{ccc}
\phi_{1}\left(p_{1}\right) & \cdots & \phi_{N}\left(p_{1}\right) \\
\vdots & \ddots & \vdots \\
\phi_{1}\left(p_{M^{\partial \Omega}}\right) & \cdots & \phi_{N}\left(p_{M^{\partial \Omega}}\right)
\end{array}\right] .
\end{gathered}
$$

and

$$
\begin{aligned}
\mathbf{M}_{1}^{\partial \Omega} & =\left[\begin{array}{ccc}
n_{1}\left(p_{1}\right) \phi_{1}\left(p_{1}\right) & \cdots & n_{1}\left(p_{1}\right) \phi_{N}\left(p_{1}\right) \\
\vdots & \ddots & \vdots \\
n_{1}\left(p_{\left.M^{\partial \Omega}\right)}\right) \phi_{1}\left(p_{M^{\partial \Omega}}\right) & \cdots & n_{1}\left(p_{M^{\partial \Omega}}\right) \phi_{N}\left(p_{\left.M^{\partial \Omega}\right)}\right)
\end{array}\right], \\
\mathbf{M}_{2}^{\partial \Omega} & =\left[\begin{array}{ccc}
n_{2}\left(p_{1}\right) \phi_{1}\left(p_{1}\right) & \cdots & n_{2}\left(p_{1}\right) \phi_{N}\left(p_{1}\right) \\
\vdots & \ddots & \vdots \\
n_{2}\left(p_{M^{\partial \Omega}}\right) \phi_{1}\left(p_{M^{\partial \Omega}}\right) & \cdots & n_{2}\left(p_{M^{\partial \Omega}}\right) \phi_{N}\left(p_{\left.M^{\partial \Omega}\right)}\right)
\end{array}\right]
\end{aligned}
$$

Taking into account the definitions of the RBF linear combinations (35), the numerical approximations for the eigenvalues are the values $E$ for which we have nonzero solutions of the overdetermined system of linear equations

$$
\left[\begin{array}{l}
\mathbf{0} \\
\mathbf{0} \\
\mathbf{0} \\
\mathbf{0} \\
\mathbf{0} \\
\mathbf{0}
\end{array}\right]=\left(\left[\begin{array}{cccc}
\mathbf{0} & \mathbf{0} & -\mathbf{M}_{2}^{\Omega} & \mathbf{M}_{1}^{\Omega} \\
\mathbf{0} & \mathbf{0} & -\mathbf{M}_{1}^{\Omega} & -\mathbf{M}_{2}^{\Omega} \\
\mathbf{M}_{2}^{\Omega} & \mathbf{M}_{1}^{\Omega} & \mathbf{0} & \mathbf{0} \\
-\mathbf{M}_{1}^{\Omega} & \mathbf{M}_{2}^{\Omega} & \mathbf{0} & \mathbf{0} \\
\mathbf{M}_{2}^{\partial \Omega} & \mathbf{M}_{1}^{\partial \Omega} & \mathbf{M}^{\partial \Omega} & \mathbf{0} \\
-\mathbf{M}_{1}^{\partial \Omega} & \mathbf{M}_{2}^{\partial} \Omega & \mathbf{0} & \mathbf{M}^{\partial \Omega}
\end{array}\right]-E\left[\begin{array}{cccc}
\mathbf{M}^{\Omega} & \mathbf{0} & \mathbf{0} & \mathbf{0} \\
\mathbf{0} & \mathbf{M}^{\Omega} & \mathbf{0} & \mathbf{0} \\
\mathbf{0} & \mathbf{0} & \mathbf{M}^{\Omega} & \mathbf{0} \\
\mathbf{0} & \mathbf{0} & \mathbf{0} & \mathbf{M}^{\Omega} \\
\mathbf{0} & \mathbf{0} & \mathbf{0} & \mathbf{0} \\
\mathbf{0} & \mathbf{0} & \mathbf{0} & \mathbf{0}
\end{array}\right]\right) \cdot\left[\begin{array}{c}
\alpha^{(1)} \\
\beta^{(1)} \\
\alpha^{(2)} \\
\beta^{(2)}
\end{array}\right] .
$$

The numerical solution of the minimization problem associated to the non-linear variational characterization is obtained directly from (3), defining the function

$$
\begin{aligned}
& \mathcal{F}\left(\alpha_{1}^{(1)}, \ldots, \alpha_{N}^{(1)}, \beta_{1}^{(1)}, \ldots, \beta_{N}^{(1)}\right) \\
& =\frac{4 \int_{\Omega}\left|\partial_{\bar{z}} u_{1}\right|^{2} d x-E^{2} \int_{\Omega}\left|u_{1}\right|^{2} d x+E \int_{\partial \Omega}\left|u_{1}\right|^{2} d s}{\int_{\Omega}\left|u_{1}\right|^{2} d x}
\end{aligned}
$$

that we minimize by a gradient type method. We refer to [2] for details about the numerical quadratures to approximate the boundary and volume integrals in the definition of $\mathcal{F}$.

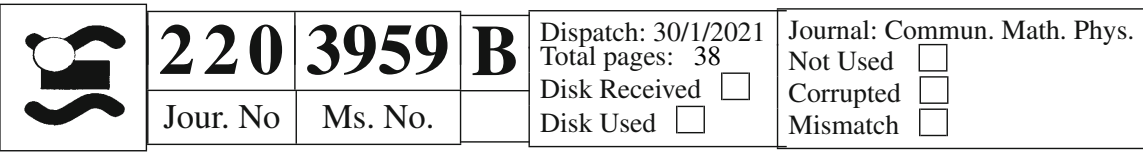


Table 1. Absolute errors of the numerical approximations for the principal eigenvalue $\lambda_{1}(\mathbb{D})$, for several choices of $\epsilon$ and $N$

\begin{tabular}{llll}
\hline & $\mathrm{N}=242$ & $\mathrm{~N}=323$ & $\mathrm{~N}=402$ \\
\hline$\epsilon=5$ & $4.45 \times 10^{-7}$ & $8.55 \times 10^{-8}$ & $1.33 \times 10^{-8}$ \\
$\epsilon=10$ & $1.30 \times 10^{-5}$ & $2.78 \times 10^{-6}$ & $4.93 \times 10^{-8}$ \\
$\epsilon=15$ & $4.92 \times 10^{-5}$ & $9.21 \times 10^{-6}$ & $1.16 \times 10^{-6}$ \\
\hline
\end{tabular}

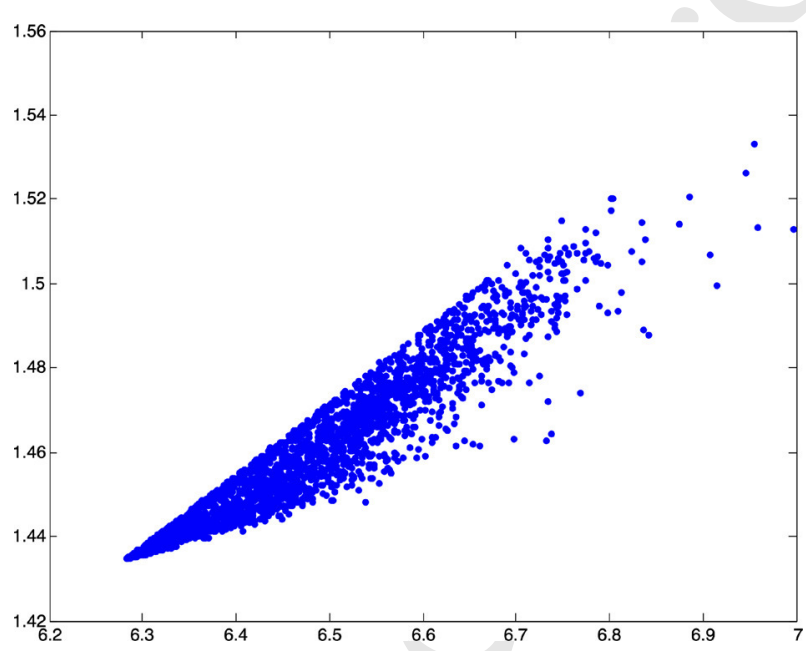

Fig. 1. Plot of the principal eigenvalue for 2500 domains (with smooth boundary) randomly generated satisfying $|\Omega|=\pi$, as a function of the perimeter

8.2. Numerical results. We start by testing our numerical algorithm for the calculation of the eigenvalues of the Dirac operator with infinite mass boundary conditions in the case of the unit disk, for which we know that the principal eigenvalue $E_{1}(\mathbb{D})$ is the smallest non-negative solution of the equation

$$
J_{0}(\mu)=J_{1}(\mu)
$$

and we have $E_{1}(\mathbb{D})=1.434695650819 \ldots$ In Table 1 we show the absolute errors of the numerical approximations for the principal eigenvalue $E_{1}(\mathbb{D})$, for several choices of $\epsilon$ and $N$ and show that the numerical method can be highly accurate, even with a moderate value of $N$.

We have computed the principal eigenvalue for 2500 domains (with smooth boundary) randomly generated satisfying $|\Omega|=\pi$. The corresponding eigenvalues are plotted in Fig. 1, as a function of the perimeter. We observe that the principal eigenvalue is minimized for the domain which also minimizes the perimeter. By the classical isoperimetric inequality it is well known that for fixed area, the perimeter is minimized by the disk. Thus, these numerical results suggest that the Faber-Krahn type inequality stated in Conjecture 1 shall hold for the Dirac operator with infinite mass boundary conditions.

Next, we present some numerical results for the minimization problem associated to the non-linear variational characterization (3). Figure 2 shows three domains (denoted by $\Omega_{1}, \Omega_{2}$ and $\left.\Omega_{3}\right)$ verifying $\left|\Omega_{i}\right|=\pi,(i=1,2,3)$ to illustrate the numerical results that we gathered. In Fig. 3 we plot $\mu^{\Omega_{i}}(E), i=1,2,3$ together with the curve $\mu^{\mathbb{D}}(E)$.

\begin{tabular}{|l|l|l|l|l|l|}
\hline $\mathbf{2} 2 \mathbf{2 0}$ & $\mathbf{3 9 5 9}$ & $\mathbf{B}$ & $\begin{array}{l}\text { Dispatch: 30/1/2021 } \\
\text { Total pages: 38 } \\
\text { Disk Received } \\
\text { Disk Used } \square\end{array}$ & $\begin{array}{l}\text { Journal: Commun. Math. Phys. } \\
\text { Not Used } \square \\
\text { Corrupted } \square \\
\text { Mismatch } \square\end{array}$ \\
\hline
\end{tabular}



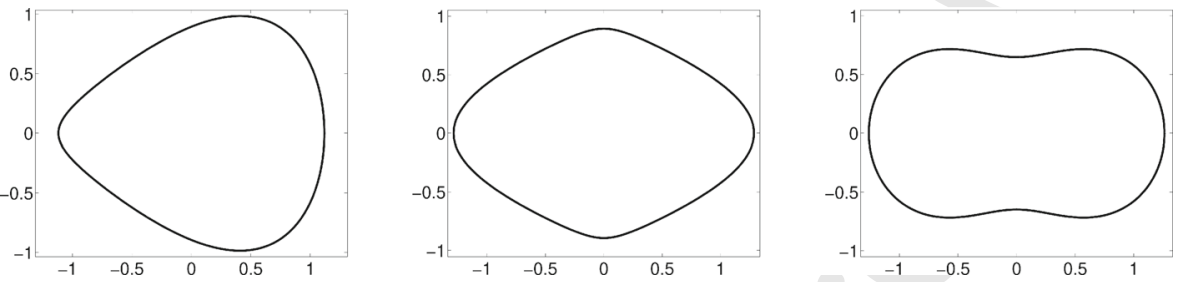

Fig. 2. Plots of domains $\Omega_{1}, \Omega_{2}$ and $\Omega_{3}$

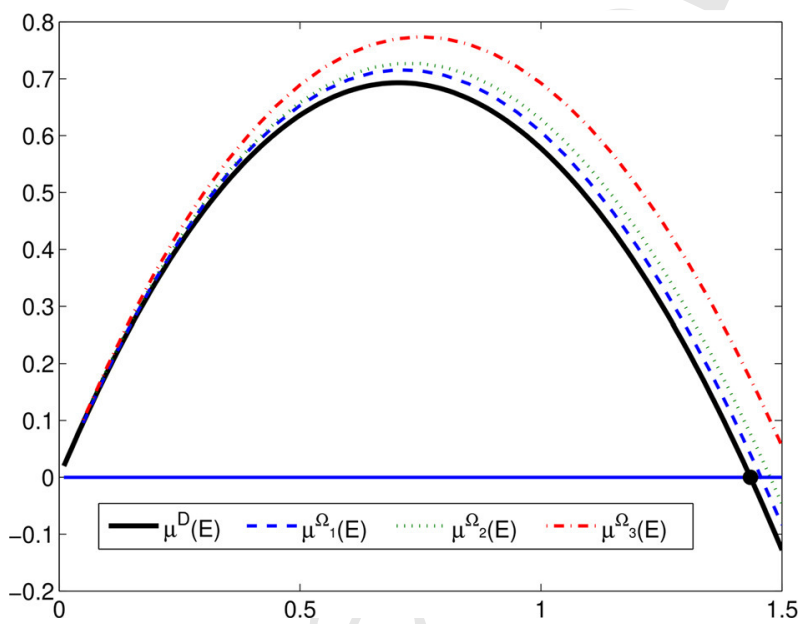

Fig. 3. Plots of $\mu^{\Omega_{i}}, i=1,2,3$, together with the curve $\mu^{\mathbb{D}}$ as a function of the spectral parameter $E>0$

We verify that for all $E>0$, we have

$$
\mu^{\Omega_{i}}(E) \geq \mu^{\mathbb{D}}(E), i=1,2,3
$$

which illustrates Conjecture 40.

Finally, Fig. 4 shows the absolute value (left plots) and argument (right plots) of a (normalized) eigenfunction associated to the principal eigenvalue of the domains $\Omega_{i}, i=$ $1,2,3$. Remark that the point of maximal modulus seems to be localized at the incenter of $\Omega_{i}$ which is in line with our choice of test function in the proof of Theorem (3). However, there is absolutely no reason for the associated eigenfunction to be real-valued and this has two consequences. First, Theorem 3 could be improved if one considers an adequate test function in the domain of the operator and not only in the form domain as we do. Second, Conjecture (1) can not be reduced to the Bossel-Daners inequality because, contrary to the Robin eigenvalue problem, there is a priori no reason for an eigenfunction to have a non-constant argument as illustrated in Fig. 4. 

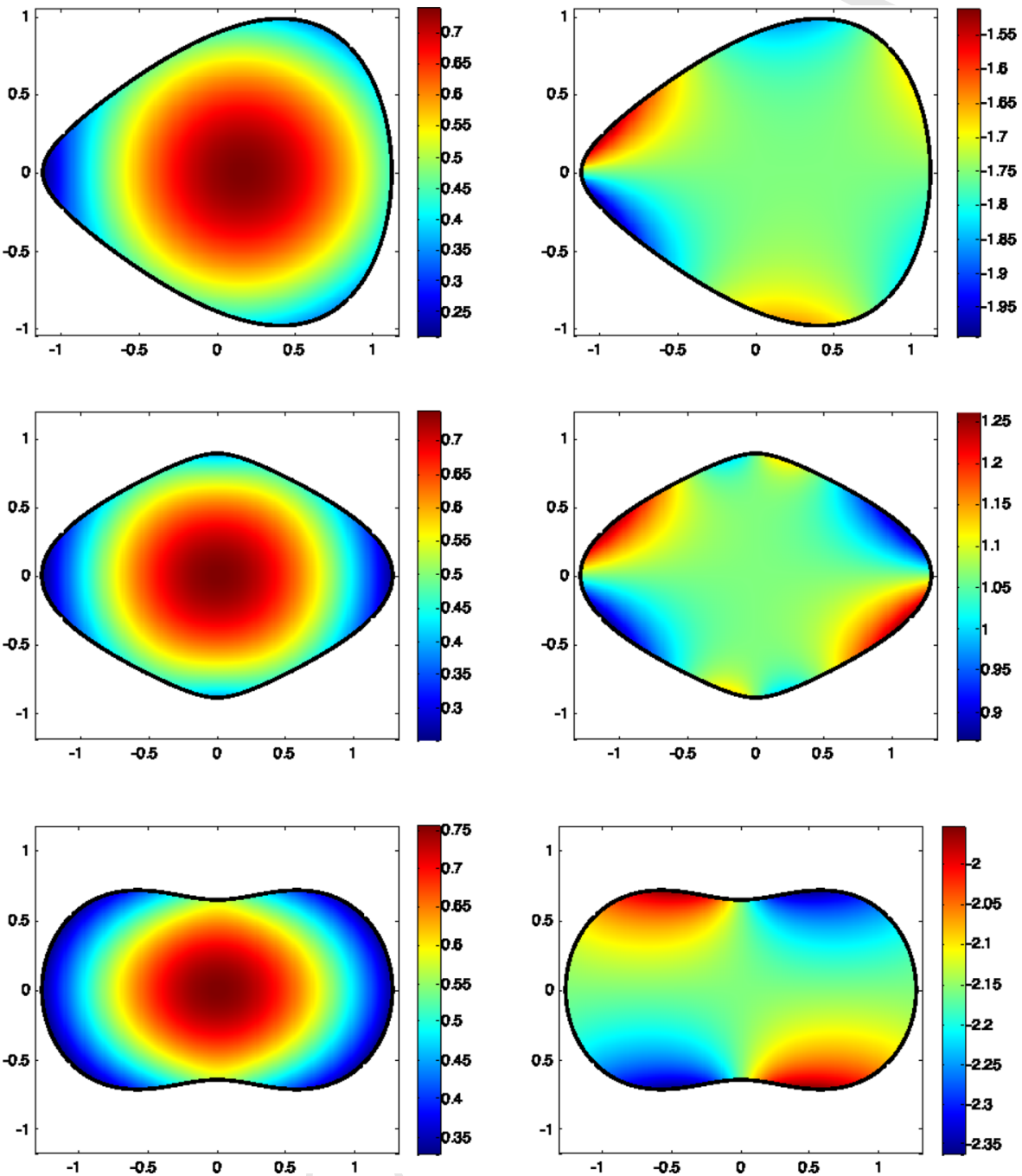

Fig. 4. Plots of the absolute value (left plots) and argument (right plots) of the eigenfunction associated to the principal eigenvalue of $\Omega_{i}, i=1,2,3$

Acknowledgements. R. D. Benguria, V. Lotoreichik and T. Ourmières-Bonafos are very grateful to the American Institute of Mathematics (AIM) for supporting their participation in the AIM workshop Shape optimization with surface interactions in 2019, where this project was initiated. The authors thank Lukas Schimmer and Jan Philip Solovej for pointing out a technical gap in an earlier version of this manuscript T. Ourmières-Bonafos thanks Nicolas Raymond for pointing out that the projectors introduced in Definition 19 are named after the famous mathematician Gábor Szegő.

Funding The work of R. D. Benguria has been partially supported by FONDECYT (Chile) projects 1160856, and 120-1055.

\begin{tabular}{|l|l|l|l|l|l|}
\hline 220 & $\mathbf{3 9 5 9}$ & $\mathbf{B}$ & $\begin{array}{l}\text { Dispatch: 30/1/2021 } \\
\text { Total pages: 38 } \\
\text { Disk Received } \\
\text { Disk Used } \square\end{array}$ & $\begin{array}{l}\text { Journal: Commun. Math. Phys. } \\
\text { Not Used } \square \\
\text { Corrupted } \\
\text { Mismatch } \square\end{array}$ \\
\hline Jour. No & Ms. No.
\end{tabular}


Publisher's Note Springer Nature remains neutral with regard to jurisdictional claims in published maps and institutional affiliations.

\section{References}

1. Agricola, I., Friedrich, T.: Upper bounds for the first eigenvalue of the Dirac operator on surfaces. J. Geom. Phys 30(1), 1-22 (1999)

2. Antunes, P.R.S.: Extremal p-Laplacian eigenvalues. Nonlinearity 32, 5087-5109 (2019)

3. Arrizabalaga, N., Le Treust, L., Mas, A., Raymond, N.: The MIT bag model as an infinite mass limit. Journal de l'École Polytechnique - Mathématiques, Tome 6, 329-365 (2019)

4. Arrizibalaga, N., Le Treust, L., Raymond, N.: On the MIT bag model in the non-relativistic limit. Commun. Math. Phys. 354, 641 (2017)

5. Arrizibalaga, N., Mas, A., Vega, L.: Shell interactions for Dirac operators. J. Math. Pures Appl. 102, 617-639 (2014)

6. Bär, C.: Lower eigenvalue estimates for Dirac operator. Math. Ann. 293, 39-46 (1992)

7. Bär, C.: Extrinsic bounds for eigenvalues of the Dirac operator. Ann. Glob. Anal. Geom. 16(2), 573-596 (1998)

8. Barbaroux, J.-M., Cornean, H.D., Le Treust, L., Stockmeyer, E.: Resolvent convergence to Dirac operators on planar domains. Ann. Henri Poincaré 20, (2019)

9. Behrndt, J., Holzmann, M., Ourmières-Bonafos, T., Pankrashkin, K.: Two-dimensional Dirac operators with singular interactions supported on closed curves. J. Funct. Anal. 279, 46 (2020)

10. Bell, S.R.: The Cauchy Transform. Potential Theory and Conformal Mapping, 2nd edn. Chapman \& Hall Book, Boca Raton (2016)

11. Benguria, R.D., Fournais, S., Van Den Bosch, H., Stockmeyer, E.: Self-adjointness of two dimensional dirac operators on domains. Ann. Henri Poincaré 18, 1371-1383 (2017)

12. Benguria, R.D, Fournais, S., Van Den Bosch, H., Stockmeyer, E.: Spectral gaps of Dirac operators describing graphene quantum dots. Math. Phys. Geom. 20, article 11 (2017)

13. Bossel, M..-H.: Membranes élastiquement liées: Extension du théorème de Rayleigh-Faber-Krahn et de l'inégalité de Cheeger. C. R. Acad. Sci. Paris Sér. I Math 302, 47-50 (1986)

14. Brezis, H.: Analyse fonctionnelle. Théorie et applications. Collection Mathématiques Appliquées pour la Maîtrise, Masson, Paris (1983)

15. Daners, D.: A Faber-Krahn inequality for Robin problems in any dimensions. Math. Ann. 335, 767-785 (2006)

16. Dolbeault, J., Esteban, M.J., Séré, E.: On the eigenvalues of operators with gaps. Application to Dirac operators. J. Funct. Anal. 174, 208-226 (2000)

17. Dolbeault, J., Esteban, M.J., Séré, E.: A variational method for relativistic computations in atomic and molecular physics. Int. J. Quantum Chem. 93, 149-155 (2003)

18. Faber, G.: Beweis, daß unter allen homogenen Membranen von gleicher Fläche und gleicher Spannung die kreisförmige den tiefsten Grundton gibt. Münch. Ber. 169-172, (1923)

19. Fefferman, C.L., Weinstein, M.: Honeycomb lattice potentials and Dirac points. J. Am. Math. Soc. 25, 1169-1220 (2012)

20. Flyer, N., Fornberg, B.: Solving PDEs with radial basis functions. Acta Numer. 24, 215-258 (2015)

21. Garnett, J.B., Marshall, D.E.: Harmonic Measure. Cambridge University Press, Cambridge (2005)

22. Griesemer, M., Siedentop, H.: A minimax principle for the eigenvalues in spectral gaps. J. Lond. Math. Soc. 60(2), 490-500 (1999)

23. Holzmann, M., Ourmières-Bonafos, T., Pankrashkin, K.: Dirac operators with Lorentz scalar interactions. Rev. Math. Phys. 30(05), 1850013 (2018)

24. Kansa, E.J.: Multiquadrics-a scattered data approximation scheme with applications to computational fluid-dynamics-I: surface approximations and partial derivative estimates. Comput. Math. Appl. 19, 127145 (1990)

25. Kato, T.: Perturbation theory for linear operators. Berlin: Springer, Reprint of the corr. print. of the 2nd ed. 1980 (1995)

26. Krahn, E.: Über eine von Rayleigh formulierte Minimaleigenschaft des Kreises. Math. Ann. 94, 97-100 (1925)

27. Le Treust, L., Ourmières-Bonafos, T.: Self-adjointness of Dirac operators with infinite mass boundary conditions in sectors. Ann. Henri Poincaré 19(5), 1465-1487 (2018)

28. Lotoreichik, V., Ourmières-Bonafos, T.: A sharp upper bound on the spectral gap for graphene quantum dots. Math. Phys. Anal. Geom. 22, 13 (2019)

\begin{tabular}{|l|l|l|l|l|l|}
\hline 220 & $\mathbf{3 9 5 9}$ & $\mathbf{B}$ & $\begin{array}{l}\text { Dispatch: 30/1/2021 } \\
\text { Total pages: 38 } \\
\text { Disk Received } \square \\
\text { Disk Used } \square\end{array}$ & $\begin{array}{l}\text { Journal: Commun. Math. Phys. } \\
\text { Not Used } \square \\
\text { Corrupted } \square \\
\text { Mismatch } \square\end{array}$ \\
\hline Jour. No & Ms. No.
\end{tabular}




\section{Communicated by R. Seiringer} Cambridge (2000) Raton (2016) (1995) 9(2), 569-600 (2019) 354-356 (1954)
29. McLean, W.: Strongly Elliptic Systems and Boundary Integral Equations. Cambridge University Press,

30. Moroianu, A., Ourmières-Bonafos, T., Pankrashkin, K.: Dirac operators on hypersurfaces as large mass limits. Comm. Math. Phys. (in press). Preprint ArXiv: 181103340

31. Ourmières-Bonafos, T., Vega, L.: A strategy for self-adjointness of Dirac operators: applications to the MIT bag model and $\delta$-shell interactions. Publ. Mat. 62(2), 397-437 (2018)

32. Pythe, K.P.: Complex Analysis. Conformal Inequalities and the Bieberbach conjecture. CRC Press, Boca

33. Raulot, S.: The Hijazi inequality on manifolds with boundary. J. Geom. Phys. 56(11), 2189-2202 (2006)

34. Saranen, J., Vainikko, G.: Periodic Integral and Pseudodifferential Equations with Numerical Approximations. Springer, Berlin (2002)

35. Sauter, S.A., Schwab, C.: Boundary Elements Methods. Translated and expanded from the, , : German Original, Springer Series in Computational Mathematics, 39, p. 2011. Springer, Berlin (2004)

36. Schimmer, L., Solovej, J.P., Tokus, S.: Friedrichs Extension and Min-Max Principle for Operators with a Gap. To appear in Ann. Henri Poincaré, preprint arXiv:1806.05206

37. Schmidt, K.M.: A remark on boundary value problems for the Dirac operator. Q. J. Math. 46(4), 509-516

38. Stockmeyer, E., Vugalter, S.: Infinite mass boundary conditions for Dirac operators. J. Spectr. Theory

39. Szegô, G.: Inequalities for certain eigenvalues of a membrane of given area. J. Ration. Mech. Anal. 3, 